\title{
CONSTRUCTING REGULAR ULTRAFILTERS FROM A MODEL-THEORETIC POINT OF VIEW
}

\author{
M. MALLIARIS AND S. SHELAH
}

AbStract. This paper contributes to the set-theoretic side of understanding Keisler's order. We consider properties of ultrafilters which affect saturation of unstable theories: the lower cofinality $\operatorname{lcf}\left(\aleph_{0}, \mathcal{D}\right)$ of $\aleph_{0}$ modulo $\mathcal{D}$, saturation of the minimum unstable theory (the random graph), flexibility, goodness, goodness for equality, and realization of symmetric cuts. We work in ZFC except when noted, as several constructions appeal to complete ultrafilters and thus assume a measurable cardinal. The main results are as follows. First, we investigate the strength of flexibility, known to be detected by non-low theories. Assuming $\kappa>\aleph_{0}$ is measurable, we construct a regular ultrafilter on $\lambda \geq 2^{\kappa}$ which is flexible but not good, and which moreover has large $\operatorname{lcf}\left(\aleph_{0}\right)$ but does not even saturate models of the random graph. This implies (a) that flexibility alone cannot characterize saturation of any theory, however (b) by separating flexibility from goodness, we remove a main obstacle to proving non-low does not imply maximal. Since flexible is precisely OK, this also shows that (c) from a set-theoretic point of view, consistently, OK need not imply good, addressing a problem from Dow (1985). Second, under no additional assumptions, we prove that there is a loss of saturation in regular ultrapowers of unstable theories, and also give a new proof that there is a loss of saturation in ultrapowers of non-simple theories. More precisely, for $\mathcal{D}$ regular on $\kappa$ and $M$ a model of an unstable theory, $M^{\kappa} / \mathcal{D}$ is not $\left(2^{\kappa}\right)^{+}$saturated; and for $M$ a model of a non-simple theory and $\lambda=\lambda^{<\lambda}, M^{\lambda} / \mathcal{D}$ is not $\lambda^{++}$-saturated. In the third part of the paper, we investigate realization and omission of symmetric cuts, significant both because of the maximality of the strict order property in Keisler's order, and by recent work of the authors on $\mathrm{SOP}_{2}$. We prove that if $\mathcal{D}$ is a $\kappa$-complete ultrafilter on $\kappa$, any ultrapower of a sufficiently saturated model of linear order will have no $(\kappa, \kappa)$-cuts, and that if $\mathcal{D}$ is also normal, it will have a $\left(\kappa^{+}, \kappa^{+}\right)$-cut. We apply this to prove that for any $n<\omega$, assuming the existence of $n$ measurable cardinals below $\lambda$, there is a regular ultrafilter $D$ on $\lambda$ such that any $D$-ultrapower of a model of linear order will have $n$ alternations of cuts, as defined below. Moreover, $D$ will $\lambda^{+}$-saturate all stable theories but will not $\left(2^{\kappa}\right)^{+}$-saturate any unstable theory, where $\kappa$ is the smallest measurable cardinal used in the construction.

\section{Contents}

Introduction

1. Background and examples

2. Definitions and conventions

3. Description of results

Received by the editors March 22, 2012 and, in revised form, September 10, 2013.

2010 Mathematics Subject Classification. Primary 03C20, 03C45, 03 E05.

The first author was partially supported by NSF grant DMS-1001666.

The second author was partially supported by grants DMS-0600940 and 1101597 and by a Gödel fellowship. The second author was also partially supported by the Israel Science Foundation grant 710/07. This is paper 996 in the second author's list of publications. 
4. Summary theorems

5. $\quad M^{\lambda} / \mathcal{D}$ is not $\left(2^{\lambda}\right)^{+}$-saturated for $T h(M)$ unstable

6. Flexible and ok ultrafilters

7. For $\kappa$ measurable, $\lambda \geq 2^{\kappa}$ there is $\mathcal{D}$ on $\lambda$ flexible but not good for $T_{r g}$

8. $M^{\lambda} / \mathcal{D}$ is not $\lambda^{++}$-saturated for $\lambda$ regular and $T h(M)$ non-simple

9. $\kappa$-complete not $\kappa^{+}$-complete implies no $(\kappa, \kappa)$-cuts

10. $\mathcal{E}$ normal and $\kappa$-complete on $\kappa$ implies a $\left(\kappa^{+}, \kappa^{+}\right)$-cut

11. Finite alternations of symmetric cuts

Appendix

References

\section{INTRODUCTION}

The motivation for our work is a longstanding, and far-reaching, problem in model theory: namely, determining the structure of Keisler's order on countable first-order theories. Introduced by Keisler in 1967, this order suggests a way of comparing the complexity of first-order theories in terms of the difficulty of producing saturated regular ultrapowers. Much of the power of this order comes from the interplay of model-theoretic structure and set-theoretic constraints. However, this interplay also contributes to its difficulty: progress requires advances in modeltheoretic analysis on the one hand, and advances in ultrapower construction on the other. Our work in this paper is of the second kind and is primarily combinatorial set theory, though the model-theoretic point of view is fundamental.

As might be expected from a problem of this scope, surprising early results were followed by many years of little progress. Results of Shelah in 19, Chapter VI (1978)] had settled Keisler's order for stable theories, as described in $\$ 1.2$ below. Apart from this work, and the result on maximality of $S P_{3}$ in [22], the problem of understanding Keisler's order on unstable theories was dormant for many years and seemed difficult.

Very recently, work of Malliaris and Shelah has led to considerable advances in the understanding of how ultrafilters and theories interact (Malliaris [12]-14, Malliaris and Shelah [16]-[17]). In particular, we now have much more information about properties of ultrafilters which have model-theoretic significance. However, the model-theoretic analysis gave little information about the relative strength of the ultrafilter properties described. In the current paper, we substantially clarify the picture. We establish various implications and non-implications between modeltheoretic properties of ultrafilters, and we develop a series of tools and constraints which help the general problem of constructing ultrafilters with a precise degree of saturation. Though we have framed this as a model-theoretically motivated project, it naturally relates to questions in combinatorial set theory, and our results answer some questions there. Moreover, an interesting and unexpected phenomenon in this paper is the relevance of measurable cardinals in the construction of regular ultrafilters; see 2.8 below.

This paper begins with several introductory sections which frame our investigations and collect the implications of the current work. We give two extended examples in \$1, the first historical, the second involving results from the current 
paper. Following this, we give definitions and fix notation in $\$ 2$ gives an overview of our main results in this paper. \$4 includes some summary theorems for our constructions. $\S \$ 511$ contain the main proofs.

In this paper, we focus on product constructions and cardinality constraints. In a related paper in preparation [15] we will focus on constructions via families of independent functions.

\section{BACKGROUND AND EXAMPLES}

In this section we give two extended examples. The first is historical; we motivate the problem of Keisler's order, i.e. of classifying first-order theories in terms of saturation of ultrapowers, by explaining the classification for the stable case. The second involves a proof from the current paper: we motivate the idea that modeltheoretic properties can give a useful way of calibrating the "strength" of ultrafilters by applying saturation arguments to prove that consistently flexible $(=\mathrm{OK})$ does not imply good.

Some definitions will be given informally; formal versions can be found in $\$ 2$ below.

1.1. Infinite and pseudofinite sets: Theories through the lens of ultrafilters. This first example is meant to communicate some intuition for the kinds of model-theoretic "complexity" to which saturation of ultrapowers is sensitive.

First, recall that questions of saturation and expressive power already arise in the two fundamental theorems of ultrapowers.

Theorem A (Łos' theorem for first-order logic). Let $\mathcal{D}$ be an ultrafilter on $\lambda \geq \aleph_{0}$, $M$ an $\mathcal{L}$-structure, $\varphi(\bar{x})$ an $\mathcal{L}$-formula, and $\bar{a} \subseteq N=M^{\lambda} / \mathcal{D}, \ell(\bar{a})=\ell(\bar{x})$. Fixing a canonical representative of each $\mathcal{D}$-equivalence class, write $\bar{a}[i]$ for the value of $\bar{a}$ at index $i$. Then

$$
N \models \varphi(\bar{a}) \Longleftrightarrow\{i \in \lambda: M \models \varphi(\bar{a}[i])\} \in \mathcal{D} .
$$

Theorem B (Ultrapowers commute with reducts). Let $M$ be an $\mathcal{L}^{\prime}$-structure, $\mathcal{L} \subseteq$ $\mathcal{L}^{\prime}$, and $\mathcal{D}$ an ultrafilter on $\lambda \geq \aleph_{0}, N=M^{\lambda} / \mathcal{D}$. Then

$$
\left.\left(M^{\lambda} / \mathcal{D}\right)\right|_{\mathcal{L}}=\left(\left.M\right|_{\mathcal{L}}\right)^{\lambda} / \mathcal{D} \text {. }
$$

That is, by itself, Theorem $\mathrm{A}$ may appear only to guarantee that $M \equiv M^{\lambda} / \mathcal{D}$. Yet combined with Theorem B] it has consequences for saturation of ultrapowers, as we now explain.

Consider the following three countable models in the language $\mathcal{L}=\{E,=\}$, for $E$ a binary relation symbol, interpreted as an equivalence relation.

- In $M_{1}, E$ is an equivalence relation with two countable classes.

- In $M_{2}, E$ is an equivalence relation with countably many countable classes.

- In $M_{3}, E$ is an equivalence relation with exactly one class of size $n$ for each $n \in \mathbb{N}$.

What variations are possible in ultrapowers of these models? That is, for $N_{i}=$ $M_{i}^{\lambda} / \mathcal{D}$, what can we say about: (a) the number of $E^{N_{i}}$-classes, (b) the possible sizes of $E^{N_{i}}$-classes, (b)' if two $E^{N_{i}}$-classes can have unequal sizes?

Observation 1.1. For any index set $I$ and ultrafilter $\mathcal{D}$ on $I$,

(1) $N_{1}=\left(M_{1}\right)^{I} / \mathcal{D}$ will have two E-classes each of size $\left|N_{1}\right|$,

(2) $N_{2}=\left(M_{2}\right)^{I} / \mathcal{D}$ will have $\left|N_{2}\right|$ E-classes each of size $\left|N_{2}\right|$. 
Proof. (1) Two classes follows by Eos' theorem, so we prove the fact about size. By Theorem $\left.\mathrm{B}\left(M_{11}^{\lambda} / \mathcal{D}\right)\right|_{\mathcal{L}}=M_{1}^{\lambda} / \mathcal{D}$, where $M_{11}$ is the expansion of $M_{1}$ to $\mathcal{L}^{\prime}=\mathcal{L} \cup\{f\}$ and $f$ is interpreted as a bijection between the equivalence classes. By Łos' theorem, $f$ will remain a bijection in $N_{1}$, but Theorem $\mathrm{B}$ means that whether we forget the existence of $f$ before or after taking the ultrapower, the result is the same.

(2) Similarly, $M_{2}$ admits an expansion to a language with a bijection $f_{1}$ between $M_{2}$ and a set of representatives of $E$-classes; a bijection $f_{2}$ between $M_{2}$ and a fixed $E$-class; and a parametrized family $f_{3}(x, y, z)$ where for each $a, b, f_{3}(x, a, b)$ is a bijection between the equivalence class of $b$ and that of $b$. So once more, by Theorems $\mathrm{A}$ and $\mathrm{B}$, the ultrapowers of $M_{2}$ are in a sense one-dimensional: if $N_{2}=M_{2}^{\lambda} / \mathcal{D}$ is an ultrapower, it will be an equivalence relation with $\left|N_{2}\right|$ classes each of which has size $\left|N_{2}\right|$.

Now for $M_{3}$, the situation is a priori less clear. Any non-principal ultrapower will contain infinite (pseudofinite) sets by Łos' theorem, but it is a priori not obvious whether induced bijections between these sets exist. It is easy to choose infinitely many distinct pseudofinite sets (let the $n$th set project a.e. to a class whose size is a power of the $n$th prime) which do not clearly admit bijections to each other in the index model $M$, nor to $M$ itself.

We have reached the frontier of what Theorem $B$ can control, and a property of ultrafilters comes to the surface:

Definition 1.2 ([20, Definition III.3.5]). Let $\mathcal{D}$ be an ultrafilter on $\lambda$, and let

$$
\mu(\mathcal{D}):=\min \left\{\prod_{t<\lambda} n_{t} / \mathcal{D}: n_{t}<\aleph_{0}, \prod_{t<\lambda} n_{t} / \mathcal{D} \geq \aleph_{0}\right\}
$$

be the minimum value of the product of an unbounded sequence of cardinals modulo $\mathcal{D}$.

Observation 1.3. Let $\mathcal{D}$ be an ultrafilter on $\lambda$, let $M_{3}$ be the model defined above, and let $N_{3}=\left(M_{3}\right)^{\lambda} / \mathcal{D}$. Then:

(1) $N_{3}$ will have $\left|N_{3}\right|$ E-classes.

(2) $E^{N_{3}}$ will contain only classes of size $\geq \mu(\mathcal{D})$, and will contain at least one class of size $\mu(\mathcal{D})$.

Proof. (1) As for the number of classes, Theorem B still applies.

(2) Choose a sequence of cardinals $n_{t}$ witnessing $\mu(\mathcal{D})$, and consider the class whose projection to the $t$ th index model has cardinality $n_{t}$.

Definition 1.2 isolates a well-defined set-theoretic property of ultrafilters, and indeed, an early theorem of the second author proved that one could vary the size of $\mu(\mathcal{D})$ :

Theorem C ([20, Shelah.VI.3.12]). Let $\mu(\mathcal{D})$ be as in Definition 1.2, Then for any infinite $\lambda$ and $\nu=\nu^{\aleph_{0}} \leq 2^{\lambda}$ there exists a regular ultrafilter $\mathcal{D}$ on $\lambda$ with $\mu(\mathcal{D})=\nu$.

Whereas the saturation of $\left(M_{1}\right)^{\lambda} / \mathcal{D}$ and of $\left(M_{2}\right)^{\lambda} / \mathcal{D}$ will not depend on $\mu(\mathcal{D})$, $N_{3}=\left(M_{3}\right)^{\lambda} / \mathcal{D}$ will omit a type of size $\leq \kappa$ of the form $\{E(x, a)\} \cup\left\{\neg x=a^{\prime}\right.$ : $\left.N_{3}=E\left(a^{\prime}, a\right)\right\}$ if and only if $\mu(\mathcal{D}) \leq \kappa$. Restricting to regular ultrafilters, so that saturation of the ultrapower does not depend on saturation of the index model but only on its theory, the same holds if we replace each $M_{i}$ by some elementarily equivalent model, and is thus a statement about their respective theories. 
This separation of theories by means of their sensitivity to $\mu(\mathcal{D})$ is, in fact, characteristic within stability. Recall that a formula $\varphi(x ; y)$ has the finite cover property with respect to a theory $T$ if for all $n<\omega$, there are $a_{0}, \ldots, a_{n}$ in some model $M \models T$ such that the set $\Sigma_{n}=\left\{\varphi\left(x ; a_{0}\right), \ldots, \varphi\left(x ; a_{n}\right)\right\}$ is inconsistent but every $n$-element subset of $\Sigma_{n}$ is consistent.

Theorem D ([20, Shelah VI.5]). Let $T$ be a countable stable theory, $M \models T$, and $\mathcal{D}$ a regular ultrafilter on $\lambda \geq \aleph_{0}$. Then:

(1) If $T$ does not have the finite cover property, then $M^{\lambda} / \mathcal{D}$ is always $\lambda^{+}$. saturated.

(2) If $T$ has the finite cover property, then $M^{\lambda} / \mathcal{D}$ is $\lambda^{+}$-saturated if and only if $\mu(\mathcal{D}) \geq \lambda^{+}$.

Thus Keisler's order on stable theories has exactly two classes, linearly ordered.

Proof. (Sketch) This relies on a characterization of saturated models of stable theories: $N$ is $\lambda^{+}$-saturated if and only if it is $\kappa(T)$-saturated and every maximal indiscernible set has size $\geq \lambda^{+}$. [This relies heavily on uniqueness of non-forking extensions: given a type $p$ one hopes to realize over some $A,|A| \leq \lambda$, restrict $p$ to a small set over which it does not fork, and use $\kappa(T)$-saturation to find a countable indiscernible sequence of realizations of the restricted type. By hypothesis, we may assume this indiscernible sequence extends to one of size $\lambda^{+}$, and by uniqueness of non-forking extensions, any element of this sequence which does not fork with $A$ will realize the type.]

Returning to ultrapowers: for countable theories, $\kappa(T) \leq \aleph_{1}$ and any nonprincipal ultrapower is $\aleph_{1}$-saturated. So it suffices to show that any maximal indiscernible set is large, and the theorem proves, by a coding argument, that this is true whenever the size of every pseudofinite set is large.

Discussion. As mentioned above, in this paper we construct ultrafilters with "model-theoretically significant properties". The intent of this example was to motivate our work by showing what "model-theoretically significant" might mean. However, the example also illustrates what kinds of properties may fit the bill. We make two general remarks.

(1) "Only formulas matter": The fact that $\mu(\mathcal{D})$ was detected by a property of a single formula, the finite cover property, is not an accident. For $\mathcal{D}$ a regular ultrafilter and $M=T$ any countable theory, $M^{\lambda} / \mathcal{D}$ is $\lambda^{+}$-saturated if and only if it is $\lambda^{+}$-saturated for $\varphi$-types, for all formulas $\varphi$ (Malliaris [11. Theorem 12]). Thus, from the point of view of Keisler's order, it suffices to understand properties of regular ultrafilters which are detected by formulas.

(2) The role of the pseudofinite structure is fundamental, reflecting the nature of the objects involved (regular ultrapowers, first-order theories). On one hand, pseudofinite phenomena can often be captured by a first-order theory. On the other, saturation of regular ultrapowers depends on finitely many conditions in each index model, since by definition regular ultrafilters $\mathcal{D}$ on $I,|I|=\lambda$ contain regularizing families, i.e. $\left\{X_{i}: i<\lambda\right\}$ such that for each $t \in I,\left|\left\{i<\lambda: t \in X_{i}\right\}\right|<\aleph_{0}$. 
1.2. Flexibility without goodness: Ultrafilters through the lens of theories. Our second example takes the complementary point of view. The following is a rich and important class of ultrafilters introduced by Keisler:

Definition 1.4 (Good ultrafilters, Keisler [4). The filter $\mathcal{D}$ on $I$ is said to be $\mu^{+}$good if every $f: \mathcal{P}_{\aleph_{0}}(\mu) \rightarrow \mathcal{D}$ has a multiplicative refinement, where this means that for some $f^{\prime}: \mathcal{P}_{\aleph_{0}}(\mu) \rightarrow \mathcal{D}, u \in \mathcal{P}_{\aleph_{0}}(\mu) \Longrightarrow f^{\prime}(u) \subseteq f(u)$ and $u, v \in \mathcal{P}_{\aleph_{0}}(\mu) \Longrightarrow$ $f^{\prime}(u) \cap f^{\prime}(v)=f^{\prime}(u \cup v)$.

We may assume the functions $f$ are monotonic (A.1).

$\mathcal{D}$ is said to be good if it is $|I|^{+}$-good.

It is natural to ask for meaningful weakenings of this notion, e.g. by requiring only that certain classes of functions have multiplicative refinements. An important example is the notion of OK, which appeared without a name in Keisler [4], was named and studied by Kunen [9] and investigated generally by Dow [3] and by Baker and Kunen [1]. We follow the definition from [3, 1.1].

Definition 1.5 (OK ultrafilters). The filter $\mathcal{D}$ on $I$ is said to be $\lambda$-OK if each monotone function $g: \mathcal{P}_{\aleph_{0}}(\lambda) \rightarrow \mathcal{D}$ with $g(u)=g(v)$ whenever $|u|=|v|$ has a multiplicative refinement $f: \mathcal{P}_{\aleph_{0}}(\lambda) \rightarrow \mathcal{D}$.

It is immediate that $\lambda^{+}$-good implies $\lambda$-OK. Though OK is an a priori weaker notion, the relative strength of $\mathrm{OK}$ and good was not clear. For instance, in [3, 3.10 and 4.7], Dow raises the problem of constructing ultrafilters which are $\lambda^{+}$-OK but not $\lambda^{+}$-good; to our knowledge, even the question of constructing $\lambda$-OK not $\lambda^{+}$good ultrafilters on $\lambda$ was open. Before discussing how a model-theoretic perspective can help with such questions, we define the main objects of interest in this paper:

Definition 1.6 (Regular filters). A filter $\mathcal{D}$ on an index set $I$ of cardinality $\lambda$ is said to be $\lambda$-regular, or simply regular, if there exists a $\lambda$-regularizing family $\left\langle X_{i}: i<\lambda\right\rangle$, which means that:

- for each $i<\lambda, X_{i} \in \mathcal{D}$, and

- for any infinite $\sigma \subset \lambda$, we have $\bigcap_{i \in \sigma} X_{i}=\emptyset$.

Equivalently, for any element $t \in I, t$ belongs to only finitely many of the sets $X_{i}$.

Now we make a translation. As Keisler observed, good regular ultrafilters can be characterized as those regular ultrafilters able to saturate any countable theory. (By " $\mathcal{D}$ saturates $T$ " we will always mean: $\mathcal{D}$ is a regular ultrafilter on the infinite index set $I, T$ is a countable complete first-order theory and for any $M \models T$, we have that $M^{I} / \mathcal{D}$ is $\lambda^{+}$-saturated, where $\lambda=|I|$.) We state this as a definition and an observation, which together say simply that the distance between consistency of a type (i.e. finite consistency, reflected by Łos' theorem) and realization of a type in a regular ultrapower can be explained by whether or not certain monotonic functions have multiplicative refinements.

Definition 1.7. Let $T$ be a countable complete first-order theory, $M \models T, \mathcal{D}$ a regular ultrafilter on $I,|I|=\lambda, N=M^{\lambda} / \mathcal{D}$. Let $p(x)=\left\{\varphi_{i}\left(x ; a_{i}\right): i<\lambda\right\}$ be a consistent partial type in the ultrapower $N$. Then a distribution of $p$ is a map $d: \mathcal{P}_{\aleph_{0}}(\lambda) \rightarrow \mathcal{D}$ which satisfies:

(1) For each $\sigma \in[\lambda]^{<\aleph_{0}}, d(\sigma) \subseteq\left\{t \in I: M \models \exists x \bigwedge\left\{\varphi_{i}\left(x ; a_{i}[t]\right): i \in \sigma\right\}\right\}$. Informally speaking, $d$ refines the Łos map.

(2) $d$ is monotonic, meaning that $\sigma, \tau \in[\lambda]^{<\aleph_{0}}, \sigma \subseteq \tau$ implies $d(\sigma) \supseteq d(\tau)$. 
(3) The set $\left\{d(\sigma): \sigma \in[\lambda]^{<\aleph_{0}}\right\}$ is a regularizing family, i.e. each $t \in I$ belongs to only finitely many elements of this set.

Observation 1.8. Let $T$ be a countable complete first-order theory, $M \models T, \mathcal{D}$ a regular ultrafilter on $\lambda$, and $N=M^{\lambda} / \mathcal{D}$. Then the following are equivalent:

(1) For every consistent partial type $p$ in $N$ of size $\leq \lambda$, some distribution $d$ of $p$ has a multiplicative refinement.

(2) $N$ is $\lambda^{+}$-saturated.

Proof. The obstacle to realizing the type is simply that, while Los' theorem guarantees each finite subset of $p$ is almost-everywhere consistent, there is no a priori reason why, at an index $t \in I$ at which $M \models \exists x \bigwedge\left\{\varphi_{i}\left(x ; a_{i}[t]\right): i \in \sigma\right\}$, $M \models \exists x \wedge\left\{\varphi_{i}\left(x ; a_{j}[t]\right): j \in \tau\right\}$, these two sets should have a common witness. The statement that $d$ has a multiplicative refinement is precisely the statement that there is, in fact, a common witness almost everywhere, in other words $t \in d(\sigma) \cap d(\tau) \Longrightarrow t \in d(\sigma \cup \tau)$. When this happens, we may choose at each index $t$ an element $c_{t}$ such that $\sigma \in[\lambda]^{<\aleph_{0}} \wedge t \in d(\sigma) \Longrightarrow M \models \bigwedge\left\{\varphi_{i}\left(c_{t}: a_{i}[t]\right): i \in \sigma\right\}$, by 1.7(1). Then by Łos' theorem and 1.7(1), $\prod_{t<\lambda} c_{t}$ will realize $p$ in $N$.

The other direction is clear (choose a realization $a$ and use Eos' theorem to send each finite subset of the type to the set on which it is realized by $a$ ).

Keisler's characterization of good ultrafilters then follows from showing that there are first-order theories which can "code" enough possible patterns to detect whether any $f: \mathcal{P}_{\aleph_{0}}(\lambda) \rightarrow \mathcal{D}$ fails to have a multiplicative refinement.

Note that first-order theories correspond naturally to monotonic functions of a certain kind (depending, very informally speaking, on some notion of the patterncomplexity inherent in the theory) and thus, were one to succeed in building ultrafilters which were able to saturate certain theories and not saturate others, this would likewise show a meaningful weakening of goodness.

In this context we mention a property which arose in the study of certain unstable simple theories, called non-low. The original definition is due to Buechler.

Definition 1.9. The formula $\varphi(x ; y)$ is called non-low with respect to the theory $T$ if in some sufficiently saturated model $M \models T$, for arbitrarily large $k<\omega$, there exists an infinite indiscernible sequence $\left\{a_{i}: i<\omega\right\}$, with $i<\omega \Longrightarrow \ell\left(a_{i}\right)=\ell(y)$, such that every $k$-element subset of

$$
\left\{\varphi\left(x ; a_{i}\right): i<\omega\right\}
$$

is consistent, but every $k+1$-element subset is inconsistent.

Here we make a second translation. Recall from Definition 1.6 above that the characteristic objects of regular filters $\mathcal{D}$ on $\lambda$ are $(\lambda)$-regularizing families, i.e. sets of the form $\left\{X_{i}: i<\lambda\right\}$ with $t \in I \Longrightarrow\left|\left\{i<\lambda: t \in X_{i}\right\}\right|=n_{t}<\aleph_{0}$. Malliaris had noticed in 12 that non-low formulas could detect the size (i.e. the non-standard integer whose $t$ th coordinate is $n_{t}$ ) of the regularizing families in $\mathcal{D}$, and thus had defined and studied the "flexibility" of a filter (Definition 1.10).

Definition 1.10 (Flexible ultrafilters, Malliaris [12, [13]). We say that the filter $\mathcal{D}$ is $\lambda$-flexible if for any $f \in I^{I} \mathbb{N}$ with $n \in \mathbb{N} \Longrightarrow n<_{\mathcal{D}} f$, we can find $X_{\alpha} \in \mathcal{D}$ for $\alpha<\lambda$ such that for all $t \in I$

$$
f(t) \geq\left|\left\{\alpha: t \in X_{\alpha}\right\}\right| .
$$


Informally, given any non-standard integer, we can find a $\lambda$-regularizing family below it.

Specifically, Malliaris had shown that if $\mathcal{D}$ is not $\lambda$-flexible, then it fails to $\lambda^{+}$saturate any theory containing a non-low formula. (Note that by Keisler's observation about good ultrafilters, any property of ultrafilters which can be shown to be detected by formulas must necessarily hold for good ultrafilters.) Moreover, there is a useful convergence. Kunen had brought the definition of "OK" filters to Malliaris' attention in 2010; " $\lambda$-flexible" and " $\lambda$-OK" are easily seen to be equivalent (Claim 6.1 below).

We now sketch the proof from $\$ 7$ below that consistently flexible need not imply good. (This paper and its sequel [15] contain at least three distinct proofs of that fact, of independent interest.) The numbering of results follows that in $₫ 7$

To begin, we use a diagonalization argument to show that saturation decays in ultrapowers of the random graph, i.e. the Rado graph (Definition 2.15 below). ("The random graph" means, from the set-theoretic point of view, that the function which fails to have a multiplicative refinement will code the fact that there are two sets $A, B$ in the final ultrapower $N,|A|=|B|=\lambda$, which are disjoint in $N$ but whose projections to the index models cannot be taken to be a.e. disjoint.)

Claim 5.2. Assume $\lambda \geq \kappa \geq \aleph_{0}, T=T_{r g}, M$ is a $\lambda^{+}$-saturated model of $T$, and $E$ is a uniform ultrafilter on $\kappa$ such that $\left|\kappa^{\kappa} / E\right|=2^{\kappa}$. Then $M^{\kappa} / E$ is not $\left(2^{\kappa}\right)^{+}$-saturated.

Note that the hypothesis of the claim will be satisfied when $E$ is regular, and also when $E$ is complete. Our strategy will be to take a product of ultrafilters $D \times E$, where $D$ is a regular ultrafilter on $\lambda$ and $E$ is an ultrafilter on $\kappa$. (The idea of taking such a product goes back at least as far as Keisler's work on ultraproducts of finite sets.) Then $D \times E$ will be regular, and if $\lambda \geq 2^{\kappa}$, it will fail to saturate the random graph, thus fail to be good. What remains is to ensure flexibility, and for this we will need $E$ to be $\aleph_{1}$-complete. In the following corollary, $\operatorname{lcf}\left(\aleph_{0}, \mathcal{D}\right)$ is the coinitiality of $\mathbb{N}$ in $(\mathbb{N},<)^{I} / \mathcal{D}$, i.e. the cofinality of the set of $\mathcal{D}$-non-standard integers.

Corollary 7.3. Let $\lambda, \kappa \geq \aleph_{0}$ and let $\mathcal{D}_{1}, E$ be ultrafilters on $\lambda, \kappa$ respectively where $\kappa>\aleph_{0}$ is measurable. Let $\mathcal{D}=\mathcal{D}_{1} \times E$ be the product ultrafilter on $\lambda \times \kappa$. Then:

(1) If $\mathcal{D}_{1}$ is $\lambda$-flexible and $E$ is $\aleph_{1}$-complete, then $\mathcal{D}$ is $\lambda$-flexible.

(2) If $\lambda \geq \kappa$ and $\operatorname{lcf}\left(\aleph_{0}, \mathcal{D}_{1}\right) \geq \lambda^{+}$, then $\operatorname{lcf}\left(\aleph_{0}, \mathcal{D}\right) \geq \lambda^{+}$, so in particular, $\mathcal{D}=D \times E$ will $\lambda^{+}$-saturate any countable stable theory.

Proof. (Sketch) (1) We first show that the following are equivalent: (i) any $\mathcal{D}$ non-standard integer projects $E$-a.e. to a $\mathcal{D}_{1}$-non-standard integer, (ii) $E$ is $\aleph_{1}$ complete. Then, since we have assumed (ii) holds, let some $\mathcal{D}$-non-standard integer $n_{*}$ be given. By (ii), for $E$-almost all $t \in \kappa, n_{*}[t]$ is $\mathcal{D}_{1}$-non-standard and by the flexibility of $\mathcal{D}_{1}$ there is a regularizing family $\left\{X_{i}^{t}: i<\lambda\right\} \subseteq \mathcal{D}_{1}$ below any such $n_{*}[t]$. Let $X_{i}=\left\{(s, t): s \in X_{i}^{t}\right\} \subseteq \mathcal{D}$. It follows that $\left\{X_{i}: i<\lambda\right\}$ is a regularizing family in $\mathcal{D}$ below $m_{*}$ and thus below $n_{*}$.

(2) From the first sentence of (1), we show that if $E$ is $\aleph_{1}$-complete and in addition $\operatorname{lcf}\left(\aleph_{0}, \mathcal{D}\right) \geq \lambda^{+}$, then the $\mathcal{D}_{1}$-non-standard integers (under the diagonal embedding) are cofinal in the $\mathcal{D}$-non-standard integers. This suffices. For the second clause, see Theorem $\mathrm{F}$ \$ below. 
Thus we obtain:

Theorem 7.4. Assume $\aleph_{0}<\kappa<\lambda=\lambda^{\kappa}, 2^{\kappa} \leq \lambda, \kappa$ measurable. Then there exists a regular ultrafilter $\mathcal{D}$ on $\lambda$ such that $\mathcal{D}$ is $\lambda$-flexible, yet for any model $M$ of the theory of the random graph, $M^{\lambda} / \mathcal{D}$ is not $\left(2^{\kappa}\right)^{+}$-saturated. Thus $\mathcal{D}$ is not good, and will fail to $\left(2^{\kappa}\right)^{+}$-saturate any unstable theory. However, $\mathcal{D}$ will $\lambda^{+}$-saturate any countable stable theory.

Note that the model-theoretic failure of saturation is quite strong, more so than simply "not good". The random graph is known to be minimum among unstable theories in Keisler's order (meaning that any regular $\mathcal{D}$ which fails to saturate the random graph will fail to saturate any other unstable theory). This is the strongest failure of saturation one could hope for given that $\operatorname{lcf}\left(\aleph_{0}, \mathcal{D}\right)$ is large; see Section 4 for details.

Theorem 7.4 has the following immediate corollary in the language of OK and good.

Corollary 1.11. Assume $\aleph_{0}<\kappa<2^{\kappa} \leq \mu_{1} \leq \mu_{2}<\lambda=\lambda^{\kappa}$ and $\kappa$ is measurable. Then there exists a regular ultrafilter $\mathcal{D}$ on $\lambda$ such that $\mathcal{D}$ is $\lambda$-flexible, thus $\lambda$-OK,

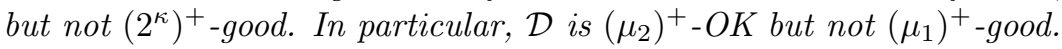

In particular, this addresses the problem raised by Dow in [3], 3.10 and 4.7, namely, the problem of constructing ultrafilters which are $\alpha^{+}$-OK and not $\alpha^{+}$good.

Discussion. The intent of this example was to show that model theory can contribute to calibrating ultrafilters. Note that in terms of determining the strength of a priori weakenings of goodness, the model-theoretic perspective has given both positive and negative results:

(1) On one hand, Theorem 7.4 applies model-theoretic arguments to show that multiplicative refinements for size-uniform functions $f: \mathcal{P}_{\aleph_{0}}(\lambda) \rightarrow \mathcal{D}$ are not enough to guarantee multiplicative refinements for all such functions.

(2) On the other hand, the second author's proof of the maximality of strict order (see Theorem $\mathrm{F}(6)$ below) does isolate an a priori weaker class of functions which have such a guarantee - namely, those corresponding to distributions of types in linear order. The set-theoretic question of why these functions suffice appears to be deep. The model-theoretic formulation of "determine a minimum such set of functions" is: determine a necessary condition for maximality in Keisler's order.

This concludes our two examples. We now fix definitions and notation, before giving a summary of our results in $\$ 3$.

\section{Definitions And COnVEntions}

This section contains background, most definitions, and conventions. Note that the definition of $\mu(\mathcal{D})$ was given in Definition [1.2, and the definitions of good, regular, and flexible filters were Definitions 1.4 1.6, and 1.10 above. (Recall that a filter is said to be $\lambda$-regular if it contains a family of $\lambda$ sets any countable number of which have empty intersection; 1.6 above.) 
Let $I=\lambda \geq \aleph_{0}$ and fix $f: \mathcal{P}_{\aleph_{0}}(\lambda) \rightarrow I$. Then $\left\{\left\{s \in I: \eta \in f^{-1}(s)\right\}: \eta<\lambda\right\}$ can be extended to a regular filter on $I$, so regular ultrafilters on $\lambda \geq \aleph_{0}$ always exist; see 2 .

Keisler proposed in 1967 [5] that saturation properties of regular ultrapowers might be used to classify countable first-order theories. His preorder $\unlhd$ on theories is often thought of as a partial order on the $\unlhd$-equivalence classes, and so known as "Keisler's order".

Definition 2.1 (Keisler [5]). Given countable theories $T_{1}, T_{2}$, say that:

(1) $T_{1} \unlhd_{\lambda} T_{2}$ if for any $M_{1} \models T_{1}, M_{2} \models T_{2}$, and $\mathcal{D}$ a regular ultrafilter on $\lambda$, if $M_{2}^{\lambda} / \mathcal{D}$ is $\lambda^{+}$-saturated, then $M_{1}^{\lambda} / \mathcal{D}$ must be $\lambda^{+}$-saturated.

(2) (Keisler's order) $T_{1} \unlhd T_{2}$ if for all infinite $\lambda, T_{1} \unlhd_{\lambda} T_{2}$.

Question 2.2. Determine the structure of Keisler's order.

The hypothesis regular justifies the quantification over all models: when $T$ is countable and $\mathcal{D}$ is regular, saturation of the ultrapower does not depend on the choice of index model.

Theorem E (Keisler [5, Corollary 2.1, p. 30]; see also Shelah [20, VI.1]). Suppose that $M_{0} \equiv M_{1}$, the ambient language is countable, and $\mathcal{D}$ is a regular ultrafilter on $\lambda$. Then $M_{0}{ }^{\lambda} / \mathcal{D}$ is $\lambda^{+}$-saturated iff $M_{1}{ }^{\lambda} / \mathcal{D}$ is $\lambda^{+}$-saturated.

More information on Keisler's order, including many examples and a summary of all known results through early 2010, may be found in the introduction to the first author's paper [13].

Definition 2.3 (Complete ultrafilters). The ultrafilter $\mathcal{E}$ on $\kappa$ is said to be $\mu$ complete if for any $\left\{X_{i}: i<\mu^{\prime}<\mu\right\} \subseteq \mathcal{E}, \bigcap\left\{X_{i}: i<\mu^{\prime}\right\} \in \mathcal{E}$.

Working with complete ultrafilters, we are obliged to make large cardinal hypotheses. We will use measurable, normal and to a lesser extent, weakly compact cardinals. Their utility for our arguments will be clear from the choice of definitions:

Definition 2.4 (Measurable, weakly compact).

(1) The uncountable cardinal $\kappa$ is said to be measurable if there is a $\kappa$-complete non-principal ultrafilter on $\kappa$.

(2) The uncountable cardinal $\kappa$ is said to be weakly compact if $\kappa \rightarrow(\kappa)_{2}^{2}$.

Fact 2.5. If $\kappa>\aleph_{0}$ is weakly compact, $n<\aleph_{0}$, and $\rho<\kappa$, then for any $\alpha:[\kappa]^{n} \rightarrow \rho$ there exists $\mathcal{U} \subseteq \kappa,|\mathcal{U}|=\kappa$ such that $\left\langle\alpha\left(\epsilon_{1}, \ldots, \epsilon_{n}\right): \epsilon_{1}<\cdots<\epsilon_{n}\right.$ from $\left.\mathcal{U}\right\rangle$ is constant.

Definition 2.6 (Normal ultrafilters). A filter $D$ on $\kappa$ is normal when, for any sequence $\left\langle A_{i}: i<\kappa\right\rangle$ with $i<\kappa \Longrightarrow A_{i} \in D$,

$$
\left\{\alpha<\kappa:(\forall j<1+\alpha)\left(\alpha \in A_{j}\right)\right\} \in D .
$$

Fact 2.7. Let $\kappa$ be a measurable cardinal. Then:

(1) There exists a normal, $\kappa$-complete, uniform ultrafilter $D$ on $\kappa$.

(2) For any $f: \kappa \rightarrow \kappa$ which is regressive on $X \in D$, there is a set $Y \in D$, $Y \subseteq X$ on which $f$ is constant. 
Discussion 2.8. An interesting and unexpected phenomenon visible in this work is the relevance of measurable cardinals, and in particular $\kappa$-complete non-principal ultrafilters, in the construction of regular ultrafilters. In the 1960s, model theorists pointed out regularity as a central property of ultrafilters, and generally concentrated on this case. Regularity ensures that saturation [of ultrapowers of models of complete countable theories] does not depend on the saturation of the index model, and that the cardinality of ultrapowers can be settled $\left(M^{I} / \mathcal{D}=M^{|I|}\right)$. Meanwhile, the construction of various non-regular ultrafilters was investigated by set theorists. However, many questions about regular ultrafilters remained opaque from the model-theoretic point of view. For example, from the point of view of Theorem $\mathrm{D}$ above, the regular ultrafilters with large $\operatorname{lcf}\left(\aleph_{0}\right)$ - a condition which implies that these ultrafilters saturate ultrapowers of stable theories - appeared to look alike. Moreover, it was not clear whether various a priori weakenings of goodness (e.g. flexible/ok) were indeed weaker. Here, in several different constructions, we combine both lines of work, using $\kappa$-complete ultrafilters to construct regular ultrafilters on $\lambda>\kappa$ with model-theoretically meaningful properties, i.e. presence or absence of some specific kind of saturation.

Definition 2.9 (Good for equality, Malliaris [14]). Let $\mathcal{D}$ be a regular ultrafilter. Say that $\mathcal{D}$ is good for equality if for any set $X \subseteq N=M^{I} / \mathcal{D},|X| \leq|I|$, there is a distribution $d: X \rightarrow \mathcal{D}$ such that $t \in \lambda, t \in d(a) \cap d(b)$ implies that $(M \models a[t]=$ $b[t]) \Longleftrightarrow(N \models a=b)$.

Definition 2.10 (Lower cofinality, $\operatorname{lcf}(\kappa, D)$ ). Let $D$ be an ultrafilter on $I$ and $\kappa$ a cardinal. Let $N=(\kappa,<)^{I} / \mathcal{D}$. Let $X \subset N$ be the set of elements above the diagonal embedding of $\kappa$. We define $\operatorname{lcf}(\kappa, D)$ to be the cofinality of $X$ considered with the reverse order.

Definition 2.11 (Product ultrafilters). Let $I_{1}, I_{2}$ be infinite sets and let $D_{1}, D_{2}$ be ultrafilters on $I_{1}, I_{2}$ respectively. Then the product ultrafilter $D=D_{1} \times D_{2}$ on $I_{1} \times I_{2}$ is defined by:

$$
X \in D \Longleftrightarrow\left\{t \in I_{2}:\left\{s \in I_{1}:(s, t) \in X\right\} \in D_{1}\right\} \in D_{2}
$$

for any $X \subseteq I_{1} \times I_{2}$.

Finally, it will be useful to have a name for functions, or relations, to which Łos' theorem applies since they are visible in an expanded language:

Definition 2.12 (Induced structure). Let $N=M^{\lambda} / \mathcal{D}$ be an ultrapower and $X \subseteq$ $N^{m}$. Say that $X$ is an induced function, or relation, if there exists a new function, or relation, symbol $P$ of the correct arity, and an expansion $M_{t}^{\prime}$ of each index model $M_{t}$ to $\mathcal{L} \cup\{P\}$, so that $P^{N} \equiv X \bmod \mathcal{D}$.

Equivalently, $X$ is the ultraproduct modulo $\mathcal{D}$ of its projections to the index models.

Definition 2.13 (Cuts in regular ultrapowers of linear orders).

(1) For a model $M$ expanding the theory of linear order, a $\left(\kappa_{1}, \kappa_{2}\right)$-cut in $M$ is given by sequences $\left\langle a_{i}: i<\kappa_{1}\right\rangle,\left\langle b_{j}: j<\kappa_{2}\right\rangle$ of elements of $M$ such that

- $i_{1}<i_{2}<\kappa_{1} \Longrightarrow a_{i_{1}}<a_{i_{2}}$,

- $j_{1}<j_{2}<\kappa_{2} \Longrightarrow b_{j_{2}}<b_{j_{1}}$

- $i<\kappa_{1}, j<\kappa_{2}$ implies $a_{i}<b_{j}$, and

- the type $\left\{a_{i}<x<b_{j}: i<\kappa_{1}, j<\kappa_{2}\right\}$ is omitted in $M$. 
(2) For $\mathcal{D}$ a (regular) ultrafilter on $I$ we define:

$$
\mathcal{C}(\mathcal{D})=\left\{\left(\kappa_{1}, \kappa_{2}\right) \in\left(\operatorname{Reg} \cap|I|^{+}\right) \times\left(\operatorname{Reg} \cap|I|^{+}\right):(\mathbb{N},<)^{I} / \mathcal{D} \text { has a }\left(\kappa_{1}, \kappa_{2}\right) \text {-cut }\right\} .
$$

Here we list the main model-theoretic properties of formulas used in this paper. For $T P_{1} / S O P_{2}$ and $T P_{2}$, see $\$ 8$. The finite cover property is from Keisler [5] and the order property, independence property, and strict order property are from Shelah [20, II.4].

Definition 2.14 (Properties of formulas). Let $\varphi=\varphi(x ; y)$ be a formula of $T$ and $M \models T$ be any sufficiently saturated model. Note that $\ell(x), \ell(y)$ are not necessarily 1. Say that the formula $\varphi(x ; y)$ has:

(1) Not the finite cover property, written $n f c p$, if there exists $k<\omega$ such that: for any $A \subseteq M$ and any set $X=\{\varphi(x ; a): a \in A\}$ of instances of $\varphi$, $k$-consistency implies consistency. (This does not depend on the model chosen.)

(2) The finite cover property, written $f c p$, if it does not have nfcp.

(3) The order property if there exist elements $a_{i}(i<\omega)$ such that for each $n<\omega$, the following partial type is consistent:

$$
\left\{\neg \varphi\left(x ; a_{i}\right): i<n\right\} \cup\left\{\varphi\left(x ; a_{j}\right): j \geq n\right\} .
$$

Formulas with the order property are called unstable.

(4) The independence property if there exist elements $a_{i}(i<\omega)$ such that for each $\sigma, \tau \in[\omega]^{<\aleph_{0}}$ with $\sigma \cap \tau=\emptyset$, the following partial type is consistent:

$$
\left\{\neg \varphi\left(x ; a_{i}\right): i \in \sigma\right\} \cup\left\{\varphi\left(x ; a_{j}\right): j \in \tau\right\} .
$$

Note that the independence property implies the order property.

(5) The strict order property if there exist elements $a_{i}(i<\omega)$ such that for all $j \neq i<\omega$

$$
\left(\exists x\left(\neg \varphi\left(x ; a_{j}\right) \wedge \varphi\left(x ; a_{i}\right)\right) \Longleftrightarrow j<i\right) .
$$

Note that (4), (5) each imply (3).

A theory $T$ is said to have the finite cover property, the order property, the independence property, or the strict order property iff one of its formulas does, and to have $n f c p$ if all of its formulas do.

The "random graph" is known to be minimum in Keisler's order among the unstable theories, and so will feature in our proofs with some regularity.

Definition 2.15. The random graph, i.e. the Rado graph, is (the unique countable model of) the complete theory in the language with equality and a binary relation $R$ axiomatized by saying that there are infinitely many elements, and that for each $n$, and any two disjoint subsets of size $n$, there is an element which $R$-connects to all elements in the first set and to none in the second set.

We conclude this section with some conventions which hold throughout the paper.

Convention 2.16 (Conventions).

- The letters $D, E, \mathcal{D}, \mathcal{E}$ are used for filters. Generally, we reserve $\mathcal{D}$ for a regular filter or ultrafilter, and $\mathcal{E}$ for a $\kappa$-complete ultrafilter where $\kappa \geq \aleph_{0}$, though this is always stated in the relevant proof. 
- Throughout, tuples of variables may be written without overlines, that is: when we write $\varphi=\varphi(x ; y)$, neither $x$ nor $y$ are necessarily assumed to have length 1.

- For transparency, all languages are assumed to be countable.

- As mentioned in $₫ 1.2$, by "D $\mathcal{D}$ saturates $T$ " we will always mean: $\mathcal{D}$ is a regular ultrafilter on the infinite index set $I, T$ is a countable complete first-order theory and for any $M \models T$, we have that $M^{I} / \mathcal{D}$ is $\lambda^{+}$-saturated, where $\lambda=|I|$.

- We will also say that the ultrafilter $D$ is "good" (or: "not good") for the theory $T$ to mean that $D$ saturates (or: does not saturate) the theory $T$.

- We reserve the word cut in models of linear order for omitted types. A partial type in a model $M$ given by some pair of sequences $\left(\left\langle a_{\alpha}: \alpha<\right.\right.$ $\left.\left.\kappa_{1}\right\rangle,\left\langle b_{\beta}: \beta<\kappa_{2}\right\rangle\right)$ with $\alpha<\alpha^{\prime}<\kappa_{1}, \beta<\beta^{\prime}<\kappa_{2} \Longrightarrow M \models a_{\alpha}<a_{\alpha^{\prime}}<$ $b_{\beta^{\prime}}<b_{\beta}$, which may or may not have a realization in $M$, is called a pre-cut. See also Definition 2.13.

\section{Description of Results}

In this section, we describe the main results of the paper.

Some notes: For relevant definitions and conventions (" $\mathcal{D}$ saturates $T$ ", "good for", "pre-cut") see $₫ 2$ above, in particular 2.16. Lists of the properties mentioned from the point of view of Keisler's order can be found in Theorems E[G, \$4, The reader unused to phrases of the form "not good for the random graph therefore not good" is referred to in $\$ 1.2$, especially following 1.6.

The first main result, Theorem 7.4, was discussed in $\$ 1.2$ above. We first show that for $E$ an ultrafilter on $\kappa$, if $E$ is $\kappa$-regular or $E$ is $\kappa$-complete, then $M^{\kappa} / E$ will not be $\left(2^{\kappa}\right)^{+}$-saturated for any $\kappa^{+}$-saturated model of $T_{r g}$, the theory of the random graph. Note that this gives a useful way of producing regular ultrafilters which are not good: for any $\lambda$-regular $\mathcal{D}_{1}$ on $\lambda$, the product ultrafilter $\mathcal{D}=\mathcal{D}_{1} \times E$ will remain regular but will not be good for $T_{r g}$ when $2^{\kappa} \leq \lambda$. We then show, as sketched above, that when $E$ is $\aleph_{1}$-complete and $\operatorname{lcf}\left(\aleph_{0}, \mathcal{D}_{1}\right)$ is large the non-standard integers of $\mathcal{D}_{1}$ will be cofinal in those of $\mathcal{D}$ (under the diagonal embedding) and thus that $\mathcal{D}$ will inherit both the large $\operatorname{lcf}\left(\aleph_{0}\right)$ and the flexibility of $\mathcal{D}_{1}$.

This yields Theorem 7.4 for any $\lambda \geq 2^{\kappa}, \kappa$ measurable, there is a regular ultrafilter on $\lambda$ which is flexible but not good, which has large $\operatorname{lcf}\left(\aleph_{0}\right)$ and thus saturates all stable theories, but does not saturate any unstable theory. In at least one sense, this is a surprising reversal. From the model-theoretic point of view, flexible ultrafilters had appeared "close" in power to ultrafilters capable of saturating any first-order theory. By Theorem 7.4, consistently flexibility cannot guarantee the saturation of any unstable theory, since the random graph is minimum among the unstable theories in Keisler's order. Thus the space between flexible and good is potentially quite large.

Discussion. In fact we obtain several different flexible-not-good theorems, including Conclusion 10.4 and a related theorem in 15. These results have other advantages, and less dramatic failures of saturation. In fact, if the stated failures of saturation can be shown to be sharp, this could be quite useful for obtaining further dividing lines within the unstable theories. 
Second, we give a new proof that there is a loss of saturation in ultrapowers of non-simple theories (Conclusion 8.6). Specifically, we show that if $M$ is a model of a non-simple theory and $\mathcal{D}$ a regular ultrafilter on $\lambda=\lambda^{<\lambda}$, then for some formula $\varphi M^{\lambda} / \mathcal{D}$ is not $\lambda^{++}$-saturated for $\varphi$-types. (The second author's book [20], VI contains a first proof of this result.) The argument uses a combinatorial principle from [7] against the definition of the tree property.

The remainder of the paper focuses on realization and omission of symmetric cuts, and here complete ultrafilters are very useful; see Discussion 2.8 above. Relatively little was known about the possible spectrum of cofinalities of cuts in ultrapowers of linear order, and our theorems here contribute to these investigations. The specific model-theoretic importance of symmetric cuts in ultrapowers has come to light in subsequent results of the authors [17, [18.

We prove in Claim 9.1 that if the ultrafilter $\mathcal{E}$ is $\kappa$-complete not $\kappa^{+}$-complete, any $\mathcal{E}$-ultrapower of a sufficiently saturated model of linear order will have no $(\kappa, \kappa)$-cuts. This is a fairly direct proof, and we also show there that if we further assume that $\mathcal{E}$ is normal, then it follows that $\mathcal{E}$ is good (though not regular; see the Appendix, e.g. A.2. We then prove, in Claim 10.1, that if $\mathcal{E}$ is $\kappa$-complete and normal on $\kappa$, then any ultrapower of a sufficiently saturated model of linear order will contain a $\left(\kappa^{+}, \kappa^{+}\right)$-cut.

Finally, we leverage these proofs for a last existence result, Theorem 11.3. There, assuming $n$ measurable cardinals below $\lambda$, we show how to take products of ultrafilters to produce any number of finite alternations of cuts in an ultrafilter on $\lambda$ with clearly described saturation properties. The proof is by downward induction. Note that if the first ultrafilter in our $(n+1)$-fold product is regular, the final ultrafilter will be regular. By a result mentioned at the beginning of this section, if the first ultrafilter is flexible with large $\operatorname{lcf}\left(\aleph_{0}\right)$ and all remaining ultrafilters are at least $\aleph_{1}$-complete, the final ultrafilter will inherit flexibility and large $\operatorname{lcf}\left(\aleph_{0}\right)$, thus be able to saturate any stable theory. By the result about loss of saturation for the random graph, it will fail to $\left(2^{\kappa}\right)^{+}$-saturate any unstable theory, where $\kappa$ is the smallest measurable cardinal used in the construction.

This completes the summary of our main results. In the appendix, we collect some easy observations and extensions of previous results relevant to these theorems.

\section{Summary theOREMS}

In this section, we state and prove several comprehensive theorems which give the picture of Keisler's order (Definition 2.1) in light of our current work. The results justify the phrase "properties of ultrafilters with model-theoretic significance". Recall our conventions in 2.16, especially with respect to "saturates" and "good". Minimum, maximum, etc. refer to Keisler's order.

The first theorem collects the currently known correspondences between properties of regular ultrafilters and properties of first-order theories.

Theorem F. In the following table, for each of the rows (1),(3),(5),(6) the regular ultrafilter $\mathcal{D}$ on $\lambda$ fails to have the property in the left column if and only if it omits a type in every formula with the property in the right column. For rows (2) and (4), left to right holds: if $\mathcal{D}$ fails to have the property on the left then it omits a type in every formula with the property on the right. 
Set theory: properties of filters

(1) $\mu(\mathcal{D}) \geq \lambda^{+}$

(2) $\operatorname{lcf}\left(\aleph_{0}, \mathcal{D}\right) \geq \lambda^{+}$

(3) good for $T_{r g}$

(4) flexible, i.e. $\lambda$-flexible

(5) good for equality

(6) good, i.e. $\lambda^{+}$-good
Model theory: properties of formulas

A. finite cover property

** B. order property

C. independence property

** D. non-low

E. $T P_{2}$

F. strict order property

Proof. (Discussion - Sketch)

(1) $\leftrightarrow$ (A) Shelah [20, VI.5]; see $\$ 1.1$ above.

$(2) \leftarrow$ (B) Shelah [20, VI.4.8]; see also Theorem A.3 below which generalizes that result.

$(3) \leftrightarrow(\mathrm{C})$ Straightforward by quantifier elimination; see [13. More generally, Malliaris [14] shows that the random graph, as the minimum non-simple theory, and $T_{f e q}$, as the minimum $T P_{2}$ theory, are in a natural sense characteristic of "independence properties" seen by ultrafilters.

$(4) \leftarrow$ (D) Malliaris [12; see $\$ 1.2$ above, or [15.

$(5) \leftrightarrow(\mathrm{E})$ Malliaris [13, $\S 6]$, which proves the existence of a minimum $T P_{2}$-theory, the theory $T_{f e q}^{*}$ of a parametrized family of independent (crosscutting) equivalence relations.

$(6) \leftrightarrow(F)$ Keisler observed that good ultrafilters can saturate any countable theory, and proved that goodness is equivalent to the saturation of certain ("versatile") formulas [5], thus establishing the existence of a maximum class in Keisler's order; see $\$ 1.2$ above. The result $(6) \leftrightarrow(\mathrm{F})$ follows from Shelah's proof in [20, VI.3] that any theory with the strict order property is maximum in Keisler's order. Thus any ultrafilter able to saturate $S O P$-types must be good, and by Keisler's observation the reverse holds. In fact, $S O P_{3}$ is known to be sufficient for maximality by [22][23], but this formulation is more suggestive here given our focus on order-types and cuts. A model-theoretic characterization of the maximum class is not known.

Remark 4.1. Moreover, by work of the authors in [17] if $\mathcal{D}$ on $\lambda$ has "treetops", i.e. it realizes a certain set of $S O P_{2}$-types, then it must realize all symmetric pre-cuts, that is, there can be no $(\kappa, \kappa)$-cuts in ultrapowers of linear order for $\kappa \leq \lambda$. So we will also be interested in the property of realizing symmetric cuts.

As rows (2) and (4) of Theorem E suggest, there are subtleties to the correspondence. If $T$ is not Keisler-maximal, then any formula $\varphi$ of $T$ with the order property has the independence property, as does any non-low formula. Yet consistently neither (4) nor (2) imply (3), as the rest of this section explains. So while we have model-theoretic sensitivity to properties (2) and (4), this is not enough for a characterization: in fact it follows from the theorems below that there is consistently no theory (and no formula $\varphi$ ) which is saturated by $\mathcal{D}$ if and only if $(2)$, or (4).

Theorem G. Using the enumeration of properties of ultrafilters from Theorem $\mathrm{F}$, we have that:

(1) is necessary and sufficient for saturating stable theories,

(2) is necessary for saturating unstable theories,

(3) is necessary and sufficient for saturating the minimum unstable theory,

(4) is necessary for saturating non-low theories,

(5) is necessary and sufficient for saturating the minimum $\mathrm{TP}_{2}$ theory, 
(6) is necessary and sufficient for saturating any Keisler-maximum theory, e.g. $T h(\mathbb{Q},<)$;

note that the identity of the maximum class is not known.

Discussion. The sources follow those of Theorem E but we make some additional remarks.

(1) Note that Shelah's proof of (1) in [20, VI.5], quoted and sketched as Theorem D 1.1 above, gives the only two known equivalence classes in Keisler's order.

(2) By Shelah [20, VI.4.8] (or Theorem A.3 below) if $\mathcal{D}$ is regular and $\operatorname{lcf}\left(\mathcal{D}, \aleph_{0}\right) \leq$ $\lambda^{+}$, then any $\mathcal{D}$-ultrapower will omit a $\lambda$-type in some unstable formula, i.e., a formula with the order property. From the set-theoretic point of view, $(2) \not \rightarrow(3)$ of Theorem 4.2 shows that $\operatorname{lcf}\left(\aleph_{0}, \mathcal{D}\right) \geq \lambda^{+}$is weaker than ensuring $\lambda^{+}$-saturation for the random graph (or equivalently, for some formula with the independence property). From the model-theoretic point of view, since any unstable theory has either the strict order property or the independence property, this gap is not visible.

(3), (5) In fact what the characterization in Malliaris [13] shows is that a necessary and sufficient condition for an ultrafilter $\mathcal{D}$ on $\lambda$ to saturate the minimum $T P_{2}$ theory is that it be "good for equality", meaning that for any set $X \subseteq N=M^{\lambda} / \mathcal{D}$, $|X|=\lambda$, there is a distribution $d: X \rightarrow \mathcal{D}$ such that $t \in \lambda, t \in d(a) \cap d(b)$ implies that $(M \models a[t]=b[t]) \Longleftrightarrow(N \models a=b)$. By contrast, saturation of the minimum unstable theory asks only that for any two disjoint sets $X, Y \subseteq M^{\lambda} / \mathcal{D}$, $|X|=|Y|=\lambda$, there is a distribution $d: X \cup Y \rightarrow \mathcal{D}$ such that for any $t \in \lambda$, $a \in X, b \in Y, t \in d(a) \cap d(b)$ implies $M \models a[t] \neq b[t]$.

(4) This was discussed in $\$ 1.2$ above. Note that by work of Shelah 21 non-simple theories have an inherent structure/randomness "dichotomy" of $T P_{1}$ versus $T P_{2}$, analogous to the structure/randomness dichotomy for unstable theories of $S O P$ versus $I P$; see 98 below. We know from 13 that any ultrafilter which saturates the minimum $T P_{2}$-theory must be flexible, however we do not know whether an ultrafilter which saturates some $S O P_{2}$ theory must be flexible.

(6) See the proof of Theorem $\mathbb{E}(6)$.

With the progress in this paper, and in other recent work of the authors, we may summarize the current picture of implications as follows.

Theorem 4.2. Let properties $(1)-(6)$ be as in Theorem $\mathrm{F}$, Assume that $\mathcal{D}$ is a regular ultrafilter on $\lambda$ (note that not all of these properties imply regularity). Then:

$(1) \leftarrow(2) \leftarrow(3) \leftarrow(5) \leftarrow(6)$, with $(1) \not \rightarrow(2)$, consistently $(2) \not \rightarrow(3),(3) \not \rightarrow(5)$, and whether (5) implies (6) is open. Moreover $(1) \leftarrow(4) \leftarrow(5) \leftarrow(6)$, where

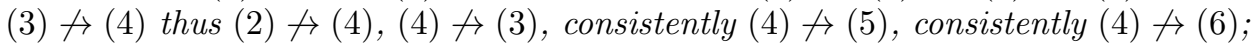
and (4) implies (2) is open.

Proof. $(6) \rightarrow(x)$ Since good ultrafilters saturate any countable theory and properties (1)-(5) are all detected by formulas via Theorem $\mathbb{F}$ property (6) implies all the others.

$(5) \rightarrow(3)$ By Theorem $\mathrm{G}$ and the fact that the random graph is minimum among unstable theories.

$(3) \rightarrow(2)$ By Theorem $\mathrm{F}$ lines (2)-(3), i.e. Shelah [20, VI.4.8].

$(2) \rightarrow$ (1) Clearly the failure of (1) implies the failure of (2).

(1) $\nrightarrow \rightarrow(2)$ Shelah [20, VI.5]; see Theorem D, \$1.2 above. 
(2) $\nrightarrow$ $\rightarrow$ (3) Consistently (assuming an $\aleph_{1}$-complete ultrafilter) by Corollary 7.3 and Theorem 7.4 below 1

$(5) \rightarrow$ (4) Malliaris [13, §6].

$(4) \rightarrow$ (1) Proved in [15, Claim 4.2].

(3) $\rightarrow \rightarrow(4)$ [16, Theorem 12.1], in light of Theorem G item (4).

(4) $\nrightarrow \rightarrow$ (3) Consistently (assuming an $\aleph_{1}$-complete ultrafilter) by Theorem 7.4 below.

(4) $\not \rightarrow(5),(6)$ We give several proofs of independent interest (each assuming the existence of an $\aleph_{1}$-complete ultrafilter): Theorem 7.4 proves $(4) \not \rightarrow(3)$ thus a fortiori $(4) \not \rightarrow(5)$, and in [15], we give a different proof that $(4) \nrightarrow \rightarrow(6)$. See also Conclusion 10.4

\section{5. $M^{\lambda} / \mathcal{D}$ IS NOT $\left(2^{\lambda}\right)^{+}$-Saturated For $T h(M)$ Unstable}

In this section and the next we prove that flexibility does not imply saturation of the random graph, and thus a fortiori that flexibility does not imply goodness for equality. This gives a proof (assuming the existence of an $\aleph_{1}$-compact ultrafilter, equivalent to the existence of a measurable cardinal) that flexible need not mean good.

Fact 5.1 ([20, Conclusion 1.13, p. 332]). If $\kappa$ is an infinite cardinal and $\mathcal{D}$ is a regular ultrafilter on $I$, then $\kappa^{I} / \mathcal{D}=\kappa^{|I|}$.

Claim 5.2. Assume $\kappa \geq \aleph_{0}, T=T_{r g}, M$ is a $\kappa^{+}$-saturated model of $T$, and $E$ is a uniform ultrafilter on $\kappa$ such that $\left|\kappa^{\kappa} / E\right|=2^{\kappa}$ (i.e. we can find a sequence $\left\langle g_{\alpha}: \alpha<2^{\kappa}\right\rangle$ of members of ${ }^{\kappa} \kappa$ such that $\left.\alpha<\beta \Longrightarrow g_{\alpha} \neq g_{\beta} \bmod E\right)$. Then $M^{\kappa} / E$ is not $\left(2^{\kappa}\right)^{+}$-saturated.

Remark 5.3. The claim is interesting when $\mathcal{D}$ is $\aleph_{1}$-complete.

Proof of [5.2. Let $\mathcal{F}=\{f: f: \kappa \times \kappa \rightarrow\{0,1\}\}$, so $|\mathcal{F}|=2^{\kappa}$, and let $\left\langle f_{\alpha}: \alpha<2^{\kappa}\right\rangle$ list $\mathcal{F}$. Let $\left\langle g_{\alpha}: \alpha<2^{\kappa}\right\rangle$ be the distinct sequence given by hypothesis. First, for each $\alpha<2^{\kappa}$, we define $\mathbf{t}_{\alpha} \in\{0,1\}$ by:

$$
\mathbf{t}_{\alpha}=1 \Longleftrightarrow\left\{i: f_{\alpha}\left(i, g_{\alpha}(i)\right)=1\right\} \notin E .
$$

Second, since $|M| \geq \kappa$, we may fix some distinguished sequence $\left\langle a_{i}: i<\kappa\right\rangle$ of elements of $M$. Let $\hat{g}_{\alpha} \in{ }^{\kappa} M$ be given by $\hat{g}_{\alpha}(i)=a_{g_{\alpha}(i)}$. Together these give a set

$$
p(x)=\left\{\left(x R \hat{g}_{\alpha} / E\right)^{\text {if } \mathbf{t}_{\alpha}}: \alpha<2^{\kappa}\right\} .
$$

We check that $p(x)$ is a consistent partial type in $M^{\kappa} / E$. It is clearly a set of formulas with parameters in $M^{\kappa} / E$, as $\hat{g}_{\alpha} / E \in M^{\kappa} / E$. Since $\alpha<\beta \Longrightarrow g_{\alpha} / E \neq$ $g_{\beta} / E \Longrightarrow \hat{g}_{\alpha} / E \neq \hat{g}_{\beta} / E$, the parameters are distinct and so the type is consistent (note that for the given sequence of parameters, any choice of exponent sequence $\left\langle\mathbf{t}_{\alpha}: \alpha<2^{\kappa}\right\rangle$ would produce a consistent partial type). Moreover, $|p|=2^{\kappa}$ again by the choice of $\left\langle g_{\alpha}: \alpha<2^{\kappa}\right\rangle$.

\footnotetext{
${ }^{1}$ Added: This is proved in ZFC for all $\lambda \geq \aleph_{2}$ in Corollary 6.6 of the authors' forthcoming paper [15].
} 
We now show that $p(x)$ is omitted in $M^{\kappa} / E$. Towards a contradiction, suppose that $h \in{ }^{\kappa} M$ were such that $h / E$ realized $p$. Let $f: \kappa \times \kappa \rightarrow\{0,1\}$ be defined by $f(i, j)=1 \Longleftrightarrow M \models h(i) R a_{j}$. Then $f \in \mathcal{F}$, hence for some $\alpha_{*}<2^{\kappa}$ we have that $f_{\alpha_{*}}=f$. Thus:

$$
\begin{aligned}
& \mathbf{t}_{\alpha_{*}}=1 \quad \text { iff } \quad M^{\kappa} / E=(h / E) R\left(\hat{g}_{\alpha_{*}} / E\right) \\
& \text { iff } \quad\left\{i<\kappa: M \models h(i) R a_{g_{\alpha_{*}}(i)}\right\} \in E \quad \text { (by Los' theorem) } \\
& \text { iff } \quad\left\{i: f\left(i, g_{\alpha_{*}}(i)\right)=1\right\} \in E \quad \text { (by the choice of } f \text { ) } \\
& \text { iff } \quad\left\{i: f_{\alpha_{*}}\left(i, g_{\alpha_{*}}(i)\right)=1\right\} \in E \quad\left(\text { as } f_{\alpha_{*}}=f\right)
\end{aligned}
$$

But by definition of the truth values $\mathbf{t}$,

$$
\mathbf{t}_{\alpha_{*}}=1 \Longleftrightarrow\left\{i: f_{\alpha_{*}}\left(i, g_{\alpha}(i)\right)=1\right\} \notin E .
$$

This contradiction completes the proof.

We state the following corollary, which will be improved in certain cases by Theorem 7.4 below. Recall the independence and strict order properties from 2.14 above.

Claim 5.4. If $\mathcal{D}$ is a $\lambda$-regular ultrafilter on $\lambda$, then $M^{\lambda} / \mathcal{D}$ is not $\left(2^{\lambda}\right)^{+}$-saturated for $M$ a model of any unstable theory.

Proof. As $T$ is unstable, it has the finite cover property. By Theorem $\mathrm{F}, M^{\lambda} / \mathcal{D}$ is not $\mu^{+}$-saturated where $\mu=\mu(\mathcal{D})$; 1.2 above. ("D is not $\aleph_{1}$-complete" suffices.) But $\mu(\mathcal{D}) \leq 2^{\lambda}$, so we are done.

Observation 5.5. The hypothesis of Claim 5.2 is satisfied when $E$ is a regular ultrafilter on $\kappa$ and when $E$ is a $\kappa$-complete ultrafilter on $\kappa$.

Remark 5.6. 5.5 is also true when $E$ is a uniform ultrafilter on $\kappa$ and $2^{\kappa}=\kappa^{+}$or $E$ is a uniform ultrafilter on $\kappa$ and $\kappa=2^{<\kappa}$.

That is, to prove Observation 5.5. we want to show that we can find a sequence $\left\langle g_{\alpha}: \alpha<2^{\kappa}\right\rangle$ of members of ${ }^{\kappa} \kappa$ so that $\alpha<\beta \Longrightarrow g_{\alpha} \neq g_{\beta} \bmod E$. When $E$ is regular, this follows from Fact 5.1. We give two proofs for the complete case, using inaccessibility of $\kappa$.

First proof of Observation [5.5 - counting functions. Suppose then that $\kappa$ is measurable, thus inaccessible. For each $\alpha<\kappa$, let $\Gamma_{\alpha}=\left\langle\gamma_{\eta}: \eta \in{ }^{\alpha} 2\right\rangle$ be a sequence of pairwise distinct ordinals $<\kappa$. For each $\eta \in{ }^{\kappa} 2$ let $g_{\eta}: \kappa \rightarrow \kappa$ be given by $g_{\eta}(\alpha)=\gamma_{\left.\eta\right|_{\alpha}}$. So $\left\{g_{\eta}: \eta \in{ }^{\kappa} 2\right\} \subseteq{ }^{\kappa} \kappa$. By construction, all we need is one point of difference to know the functions diverge: $\eta \neq \nu \in{ }^{\kappa} 2, \eta(\beta) \neq \nu(\beta) \Longrightarrow\{\alpha<\kappa$ : $\left.g_{\eta}(\alpha)=g_{\nu}(\alpha)\right\} \subseteq\{\alpha: \alpha<\beta\}=\emptyset \bmod E$ as $E$ is uniform.

Second proof of Observation 5.5 - realizing types. Suppose then that $\kappa$ is measurable, thus inaccessible. So we may choose $M,|M|=\kappa$ to be a $\kappa$-saturated model of the theory of the random graph. To show that $\left|M^{\kappa} / E\right| \geq 2^{\kappa}$, it will suffice to show that $2^{\kappa}$-many distinct types over the diagonal embedding of $M$ in the ultrapower $N$ are realized. Let $p(x)=\left\{x R f_{\alpha}^{0} \wedge \neg x R f_{\alpha}^{1}: \alpha<\kappa\right\}$ be such a type, with each $f_{\alpha}^{i}={ }^{\kappa}\{m\}$ for some $m \in M$ and of course $\alpha, \beta<\kappa \Longrightarrow f_{\alpha}^{0} \neq f_{\beta}^{1}$. For each $t \in \kappa$, let $p_{t}(x)=\left\{x R f_{\alpha}^{0}(t) \wedge \neg x R f_{\alpha}^{1}(t): \alpha<t\right\}$. Note that since the elements $f_{\alpha}^{i}$ are constant, for each $t \in \kappa$ we have that $p_{t}(x)$ is a consistent partial type in $M$. Choose a new element $h \in{ }^{\kappa} M$ so that $t \in \kappa$ implies $h(t)$ satisfies $p_{t}(x)$ in $M$. By 
the saturation of $M$, some such $h$ exists. By uniformity of $E, h$ realizes the type $p(x)$, that is, for $\alpha<\kappa$,

$$
\left|\kappa \backslash\left\{t \in \kappa: M \models h(t) R f_{\alpha}^{0}(t) \wedge \neg h(t) R f_{\alpha}^{1}(t)\right\}\right| \leq \alpha<\kappa .
$$

As no such $h$ can realize two distinct types over $M$ in $N$, we are finished.

Discussion 5.7. We may wonder: for $\mathcal{D}$ an ultrafilter on $\lambda, M$ saturated enough, $T=T h(M)$, how saturated is $M^{\lambda} / \mathcal{D}$ ? How locally saturated? Let $\kappa=\kappa(\mathcal{D})$ be the completeness of $\mathcal{D}$. In questions of saturation, we naturally assume $|T|<\kappa(\mathcal{D})$, or that $\mathcal{D}$ is $(|T|, \kappa)$-good.

Claim 5.8 is a result addressing 5.7. Further work in this direction appears in a paper of the authors in preparation.

Claim 5.8. Suppose that:

(1) $\mathcal{D}$ is an ultrafilter on $\lambda$.

(2) $\mathcal{D}$ is $\kappa$-complete not $\kappa^{+}$-complete (so $\kappa$ is measurable).

(3) $M$ is a $\kappa$-universal model (which means that every type with $\leq \kappa$ formulas and $\leq \kappa$ variables, but no parameters, is realized in $M$; if $\kappa \geq|\tau(M)|$, this means every $N \equiv M$ of cardinality $\leq \kappa$ is elementarily embeddable in $M)$.

(4) $T h(M)$ is independent, i.e. some formula has the independence property.

Then $M^{\lambda} / \mathcal{D}$ is not $\left(\mu^{*}\right)^{+}$-saturated, where

$$
\begin{aligned}
\mu^{*}=\mu^{*}(\mathcal{D})=\min \left\{\prod_{i<\lambda} \kappa_{i} / \mathcal{D}: \bigwedge_{i} \kappa_{i}<\kappa \text { and } \prod_{i} \kappa_{i} / \mathcal{D} \geq \kappa\right. \\
\left.\quad \text { and } \prod_{i} 2^{\kappa_{i}} / \mathcal{D}<2^{\prod_{i} \kappa_{i} / \mathcal{D}}\right\} .
\end{aligned}
$$

Remark 5.9. (a) If $\mathcal{D}$ is $(\lambda, \kappa)$-regular, then there is such $\mu^{*}(\mathcal{D})$ and it is $\leq 2^{\lambda}$, as $2^{\lambda}=\prod_{i} \kappa_{i} / \mathcal{D}$ for some $\kappa_{i}<\kappa$. Otherwise, it is not immediately clear whether $\mu^{*}$ is well defined. (b) In 5.8, we could just use $<\kappa$-universal.

Proof of 5.8. Let $\bar{\kappa}=\left\langle\kappa_{i}: i\langle\lambda\rangle\right.$ be a sequence witnessing $\mu^{*}(\mathcal{D})$, i.e. $\prod_{i} \kappa_{i} / \mathcal{D}=$ $\mu^{*}(\mathcal{D})$. Without loss of generality, $M$ is a model of the random graph, so $\tau(M)=$ $\{R\}$ and $x R y$ has the independence property 2 For $i<\alpha<\kappa$, let $a_{\alpha, i} \in M$ be such that $\left\langle a_{\alpha, i} R x: i<\alpha\right\rangle$ is an independent sequence of formulas, using the $(<\kappa)$ universality of $M$. For each $i$, choose $A_{i} \subseteq M, A_{i}=\left\{a_{\kappa_{i}, \epsilon}: \epsilon<\kappa_{i}\right\}$. We then choose $B_{i} \subseteq M,|B|=2^{\kappa_{i}}$ such that for every $A \subseteq A_{i}$ there is $b=b_{A}^{i} \in B_{i}$ such that $(\forall a \in A)\left(a R^{M} b \Longleftrightarrow a \in A\right)$. Let $\tau^{*}=\{R, P, Q\}$ where $P, Q$ are unary predicates. Let $M_{i}$ be the $\tau_{*}$-model expanding $M$ with $P^{M_{i}}=A_{i}, Q^{M_{i}}=B_{i}$. Let $N=M^{\lambda} / \mathcal{D}, N_{*}=\prod_{i<\lambda} M_{i} / \mathcal{D}$, so $N=N_{*}\left\lceil\tau\right.$. Let $A=P^{N_{*}}$, so $|A|=\mu^{*}$.

Now we claim there is $A^{\prime} \subseteq A$ such that no $b \in N$ realizes $p(x)=\{a R x: a \in$ $\left.A^{\prime}\right\} \cup\left\{\neg a R x: a \in A \backslash A^{\prime}\right\}$, which is a consistent partial type in $N$ (e.g. by Łos' theorem, since $\left\{x R a: a \in P^{M_{i}}\right\}$ is independent in each $M_{i}$ ). First, notice that it suffices to show there is no realization $b \in Q^{N_{*}}$, since for every $b_{1} \in N$ there is $b_{2} \in Q^{N_{*}}$ such that $a \in P^{N} \Longrightarrow a R^{N} b_{1} \equiv a R^{N} b_{2}$ [by Los' theorem and the choice of $M_{i}$. Second, $\left|\left\{A^{\prime}: A^{\prime} \subseteq A\right\}\right|=2^{\left|P^{N_{*}}\right|}=\prod_{i} \kappa_{i} / \mathcal{D}$, whereas the number of $b \in Q^{N_{*}}$ is $\left|Q^{N_{*}}\right|=\prod_{i} 2^{\kappa_{i}} / \mathcal{D}$. Moreover, each such $b$ determines an $A^{\prime}$ uniquely. But by the choice of $\left\langle\kappa_{i}: i<\lambda\right\rangle,\left|Q^{N_{*}}\right|<2^{\left|P^{N_{*}}\right|}$. This completes the proof.

\footnotetext{
${ }^{2}$ It is straightforward to translate omission of a type in the random graph to omission of a type in a formula with the independence property; see e.g. [13, §5].
} 


\section{Flexible and oK ultrafilters}

In this section we verify that "OK" may be substituted for "flexible" in our theorems below. We repeat here thanks to Kunen for suggesting the connection to the first author. The direction 6.1 (2) $\rightarrow(1)$ is in Malliaris' paper [13, Claim 8.4].

Claim 6.1. Suppose that $\mathcal{D}$ is an $\aleph_{1}$-incomplete ultrafilter on $I$. Then the following are equivalent.

(1) $\mathcal{D}$ is $\lambda$-O.K.

(2) $\mathcal{D}$ is $\lambda$-flexible.

Proof. (1) $\rightarrow(2)$ Let $M$ be given with $(\mathbb{N},<) \preceq M$ and let $h_{0} \in{ }^{I} M$ be any $\mathcal{D}$ non-standard integer. Let $\left\{Z_{n}: n<\omega\right\} \subseteq \mathcal{D}$ witness that $\mathcal{D}$ is $\aleph_{1}$-incomplete. Without loss of generality, $n<n^{\prime} \Longrightarrow Z_{n} \supseteq Z_{n^{\prime}}$. Let $h_{1} \in{ }^{I} \mathbb{N}$ be given by $h_{1}(t)=\max \left\{n: t \in Z_{n}\right\}$. Define $h \in{ }^{I} \mathbb{N}$ by

$$
h(t)=\min \left\{h_{0}(t), h_{1}(t)\right\} .
$$

Then for each $n \in \mathbb{N}, X_{n}:=\{t: h(t) \geq n\} \in \mathcal{D}$ and $X_{n} \subseteq Z_{n}$, thus $\bigcap\left\{X_{n}: n \in\right.$ $\mathbb{N}\}=\emptyset$.

Define a function $f: \mathcal{P}_{\aleph_{0}}(\lambda) \rightarrow \mathcal{D}$ by $f(u)=X_{|u|}$. As $\mathcal{D}$ is $\lambda$-OK, we may choose $g$ to be a multiplicative refinement of $f$, and consider $\mathbf{Y}=\left\{Y_{i}: i<\lambda\right\}$ given by $Y_{i}=g(\{i\})$.

First, we verify that $\mathbf{Y}$ is a regularizing family, by showing that each $t \in I$ can only belong to finitely many elements of Y. Given $t \in I$, let $m=h(t)+1<\omega$, so $t \notin X_{m}$. Suppose there were $i_{1}<\cdots<i_{m}<\lambda$ such that $t \in g\left(\left\{i_{1}\right\}\right) \cap \cdots \cap$ $g\left(\left\{i_{m}\right\}\right)$. As $g$ is multiplicative and refines $f$, this would imply $t \in g\left(\left\{i_{1}, \ldots, i_{m}\right\}\right) \subseteq$ $f\left(\left\{i_{1}, \ldots, i_{m}\right\}\right)=X_{m}$, a contradiction. Thus $\mathbf{Y}$ is a regularizing family. Moreover, as $t$ was arbitrary, we have shown that

$$
|\{i<\lambda: t \in g(\{i\})\}| \leq h(t) \leq h_{0}(t)
$$

and thus that $\mathbf{Y}$ is a regularizing family below $h_{0}$. As $h_{0}$ was an arbitrary nonstandard integer, this completes the proof.

$(2) \rightarrow(1)$ Let $f: \mathcal{P}_{\aleph_{0}}(\lambda) \rightarrow \mathcal{D}$ be such that $|u|=|v| \Longrightarrow f(u)=f(v)$, and we will construct a multiplicative refinement for $f$. Let $\left\langle Z_{n}: n\langle\omega\rangle\right.$ witness the $\aleph_{1}$-incompleteness of $\mathcal{D}$, and as before, we may assume $n<n^{\prime} \Longrightarrow Z_{n} \supseteq Z_{n^{\prime}}$. For each $t \in I$, let $\rho \in{ }^{I} \mathbb{N}$ be given by $\rho(t)=\max \left\{n \in \mathbb{N}: t \in f(n) \cap Z_{n}\right\}$, which is well defined by the choice of the $Z_{n}$. Now for each $m \in \mathbb{N}$, recalling that $m$ is an element of $[\lambda]^{m}$ via the convention $m=\{0, \ldots, m-1\}$,

$$
\{t \in I: \rho(t)>m\} \supseteq \bigcap\left\{f(n) \cap Z_{n}: n \leq m\right\} \in \mathcal{D}
$$

so $\rho$ is $\mathcal{D}$-non-standard. Applying the hypothesis of flexibility, let $\left\{Y_{i}: i<\lambda\right\}$ be a $\lambda$-regularizing family below $\rho$. Let $g: \mathcal{P}_{\aleph_{0}}(\lambda) \rightarrow \mathcal{D}$ be given by $g(\{i\})=f(\{i\}) \cap Y_{i}$ and for $|u|>1, g(u)=\bigcap\{g(\{i\}): i \in u\}$. Thus $g$ is multiplicative, by construction. Let us show that it refines $f$. Given any $n<\omega$ and $i_{1}<\cdots<i_{n}<\lambda$, observe that by definition of "below $\rho$ " we have $t \in Y_{i_{1}} \cap \cdots \cap Y_{i_{n}} \Longrightarrow \rho(t) \geq n$. Applying this fact and the definitions of $g$ and $f$,

$g\left(\left\{i_{1}, \ldots, i_{n}\right\}\right) \subseteq \bigcap\left\{Y_{i_{j}}: 1 \leq j \leq n\right\} \subseteq\{t \in I: \rho(t) \geq n\} \subseteq f(n)=f\left(\left\{i_{1}, \ldots, i_{n}\right\}\right)$,

thus $g$ refines $f$, which completes the proof. 


\section{For $\kappa$ Measurable, $\lambda \geq 2^{\kappa}$ There is $\mathcal{D}$ ON $\lambda$ FleXible BUT NOT GOOD FOR $T_{r g}$}

We begin by characterizing when flexibility is preserved under products of ultrafilters (Definition 2.11). The first observation says that $\lambda$-flexibility of the first ultrafilter ensures there are $\lambda$-regularizing families in $\mathcal{D}$ below certain non-standard integers, namely those which are a.e. $\mathcal{D}_{1}$-non-standard.

Observation 7.1. Let $\lambda, \kappa \geq \aleph_{0}$ and let $\mathcal{D}_{1}, E$ be ultrafilters on $\lambda, \kappa$ respectively. Let $\mathcal{D}=\mathcal{D}_{1} \times E$ be the product ultrafilter on $\lambda \times \kappa$. Suppose that we are given $n_{*} \in{ }^{\lambda \times \kappa} \mathbb{N}$ such that:

(1) $n \in \mathbb{N} \Longrightarrow\left\{(s, t) \in \lambda \times \kappa: n_{*}(s, t)>n\right\} \in \mathcal{D}$, i.e. $n_{*}$ is $\mathcal{D}$-non-standard.

(2) $N:=\left\{t \in \kappa: n \in \mathbb{N} \Longrightarrow\left\{s \in \lambda: n_{*}(s, t)>n\right\} \in \mathcal{D}_{1}\right\} \in E$,

i.e. E-almost all of its projections are $\mathcal{D}_{1}$-non-standard.

Then $(a) \Longrightarrow(b)$, where:

(a) $\mathcal{D}_{1}$ is $\lambda$-flexible;

(b) there is a regularizing set $\left\langle X_{i}: i<\lambda\right\rangle \subseteq \mathcal{D}$ below $n_{*}$,

i.e. such that for all $(s, t) \in \lambda \times \kappa,\left|\left\{i<\lambda:(s, t) \in X_{i}\right\}\right| \leq n_{*}(s, t)$.

Proof. For each $t \in N$, let $\left\langle X_{i}^{t}: i<\lambda\right\rangle \subseteq \mathcal{D}_{1}$ be a regularizing family below $n_{*}(-, t)$, that is, such that for each $s \in \lambda,\left|\left\{i<\lambda: s \in X_{i}^{t}\right\}\right| \leq n_{*}(s, t)$. Such a family is guaranteed by the $\lambda$-flexibility of $\mathcal{D}_{1}$ along with the definition of $N$, since the latter ensures that $n_{*}(-, t) \in{ }^{\lambda} \lambda$ is $\mathcal{D}_{1}$-non-standard. Now define $\left\langle X_{i}: i<\lambda\right\rangle$ by $X_{i}=\left\{(s, t): s \in X_{i}^{t}\right\}$. We verify that:

- $\left\langle X_{i}: i<\lambda\right\rangle \subseteq \mathcal{D}$, as $\left\{t \in \kappa:\left\{s \in \lambda:(s, t) \in X_{i}\right\} \in \mathcal{D}_{1}\right\} \supseteq N$ and $N \in E$ by hypothesis.

- $\left\langle X_{i}: i<\lambda\right\rangle$ is below $n_{*}$, since for each $(s, t) \in \lambda \times \kappa$, $\left|\left\{i:(s, t) \in X_{i}\right\}\right|=\left|\left\{i:(s, t) \in X_{i}^{t}\right\}\right| \leq n_{*}(s, t)$ by construction.

This completes the proof.

The next claim shows that $\mathcal{D}$-non-standard integers project $E$-a.e. to $\mathcal{D}_{1}$-nonstandard integers precisely when the second ultrafilter $E$ is at least $\aleph_{1}$-complete.

Claim 7.2. Let $\lambda, \kappa \geq \aleph_{0}$ and let $\mathcal{D}_{1}, E$ be uniform ultrafilters on $\lambda, \kappa$ respectively. Let $\mathcal{D}=\mathcal{D}_{1} \times E$ be the product ultrafilter on $\lambda \times \kappa$. Then the following are equivalent.

(1) If $n_{*} \in{ }^{\lambda \times \kappa} \mathbb{N}$ is such that $n \in \mathbb{N} \Longrightarrow\left\{(s, t) \in \lambda \times \kappa: n_{*}(s, t)>n\right\} \in \mathcal{D}$, then $N \in E$ where $N:=\left\{t \in \kappa: n \in \mathbb{N} \Longrightarrow\left\{s \in \lambda: n_{*}(s, t)>n\right\} \in \mathcal{D}_{1}\right\}$.

(2) $E$ is $\aleph_{1}$-complete.

Proof. (1) $\rightarrow$ (2) Suppose $E$ is not $\aleph_{1}$-complete, so it is countably incomplete and we can find $\left\langle X_{n}: n\langle\omega\rangle \subseteq E\right.$ such that $\left.\bigcap\left\{X_{n}: n \in \omega\right\rangle\right\}=\emptyset \bmod \mathcal{D}$. Without loss of generality, $n<\omega \rightarrow X_{n+1} \subsetneq X_{n}$. Let $n_{*} \in{ }^{\lambda \times \kappa} \mathbb{N}$ be given by:

$$
t \in \kappa \wedge t \in X_{n} \backslash X_{n+1} \Longrightarrow n_{*}(-, t)={ }^{\lambda}\{n\} .
$$

Then $n_{*}$ is $\mathcal{D}$-non-standard but its associated set $N$ is empty (as a subset of $\kappa$, so a fortiori empty modulo $\mathcal{D}$ ).

$(2) \rightarrow(1)$ Suppose on the other hand that $E$ is $\aleph_{1}$-complete, and let some $\mathcal{D}$ non-standard $n_{*}$ be given. For each $n \in \mathbb{N}$, define $X_{n}=\left\{t \in \kappa:\left\{s \in \lambda: n_{*}(s, t)>\right.\right.$ $\left.n\} \in \mathcal{D}_{1}\right\}$. Then by completeness, $N \supseteq \bigcap\left\{X_{n}: n \in \mathbb{N}\right\} \in E$. 
Corollary 7.3. Let $\lambda, \kappa \geq \aleph_{0}$ and let $\mathcal{D}_{1}, E$ be ultrafilters on $\lambda, \kappa$ respectively where $\kappa>\aleph_{0}$ is measurable. Let $\mathcal{D}=\mathcal{D}_{1} \times E$ be the product ultrafilter on $\lambda \times \kappa$. Then:

(1) If $\mathcal{D}_{1}$ is $\lambda$-flexible and $E$ is $\aleph_{1}$-complete, then $\mathcal{D}$ is $\lambda$-flexible.

(2) If $\lambda \geq \kappa$ and $\operatorname{lcf}\left(\aleph_{0}, \mathcal{D}_{1}\right) \geq \lambda^{+}$, then $\operatorname{lcf}\left(\aleph_{0}, \mathcal{D}\right) \geq \lambda^{+}$, so in particular, $\mathcal{D}=D \times E$ will $\lambda^{+}$-saturate any countable stable theory.

Proof. (1) By Claim 7.2 and Observation 7.1

(2) Let us show that the $\mathcal{D}_{1}$-non-standard integers are cofinal in the $\mathcal{D}$-nonstandard integers. Let $M=(\mathbb{N},<)^{\lambda} / \mathcal{D}_{1}, N=M^{\kappa} / E$. Let $n_{*} \in N$ be $\mathcal{D}$-nonstandard. By Claim 7.2, the set $X=\left\{t \in \kappa: n_{*}(t)\right.$ is a $\mathcal{D}_{1}$-non-standard element of $M\} \in E$. Since $\operatorname{lcf}\left(\aleph_{0}, \mathcal{D}_{1}\right) \geq \lambda^{+}>\kappa$, there is $m_{*} \in M$ which is $\mathcal{D}_{1}$-non-standard and such that $t \in X \Longrightarrow M \models n_{*}(t)>m_{*}$. Then the diagonal embedding of $m_{*}$ in $N$ will be $\mathcal{D}$-non-standard but below $n_{*}$, as desired. The statement about stable theories follows by $\$ 4$ Theorem $\mathrm{G}$ and Theorem $4.2(2) \rightarrow(1)$.

Theorem 7.4. Assume $\aleph_{0}<\kappa<\lambda, 2^{\kappa} \leq \lambda, \kappa$ measurable. Then there exists a regular ultrafilter $\mathcal{D}$ on $\lambda$ such that $\mathcal{D}$ is $\lambda$-flexible, yet for any model $M$ of the theory of the random graph, $M^{\lambda} / \mathcal{D}$ is not $\left(2^{\kappa}\right)^{+}$-saturated. However, $\mathcal{D}$ will $\lambda^{+}$-saturate any countable stable theory.

A fortiori, $\mathcal{D}$ is neither good nor good for equality, and it will fail to $\left(2^{\kappa}\right)^{+}$. saturate any unstable theory.

Proof. Let $\mathcal{E}$ be a uniform $\aleph_{1}$-complete ultrafilter on $\kappa$. Let $\mathcal{D}_{1}$ be any $\lambda$-flexible (thus, $\lambda$-regular) ultrafilter on $\lambda$, e.g. a regular $\lambda^{+}$-good ultrafilter on $\lambda$. Let $\mathcal{D}=\mathcal{D}_{1} \times \mathcal{E}$ be the product ultrafilter on $I=\lambda \times \kappa$. Then $|I|=\lambda$, and we have that $\mathcal{D}$ is $\lambda$-flexible, thus regular, by Corollary $7.3(1) . \mathcal{D}$ saturates countable stable theories by 7.3 (2).

Since for any model $M$,

$$
\prod_{\lambda \times \kappa} M /\left(\mathcal{D}_{1} \times \mathcal{E}\right) \cong \prod_{\kappa}\left(\prod_{\lambda} M / \mathcal{D}_{1}\right) / \mathcal{E},
$$

the right hand side shows, by Claim 5.2 , that the resulting ultrafilter will not $\left(2^{\kappa}\right)^{+}$saturate the random graph.

Finally, the "a fortiori" clause holds by Theorem 4.2

Corollary 7.5. In the construction just given, by Claim $7.2, \operatorname{lcf}\left(\aleph_{0}, \mathcal{D}\right)=$ $\operatorname{lcf}\left(\aleph_{0}, \mathcal{D}_{1}\right) \geq \lambda^{+}$since $\mathcal{D}_{1}$ is $\lambda^{+}$-good and the non-standard integers of $\mathcal{D}_{1}$ are cofinal in the non-standard integers of $\mathcal{D}$. Thus consistently, a regular ultrafilter on $\lambda>\kappa$ may have large lower cofinality of $\aleph_{0}$ while failing to $\left(2^{\kappa}\right)^{+}$-saturate the random graph.

By [20, VI.4], a necessary condition for a regular ultrafilter $\mathcal{D}$ on $\lambda$ to saturate some unstable theory is that $\operatorname{lcf}\left(\aleph_{0}, \mathcal{D}\right) \geq \lambda^{+}$; Corollary 7.5 shows it is not sufficient.

\section{8. $M^{\lambda} / \mathcal{D}$ IS NOT $\lambda^{++}$-SATURATEd FOR $\lambda$ REgular And $T h(M)$ NON-Simple}

In this section we prove that there is a loss of saturation in ultrapowers of nonsimple theories. As mentioned above, this is a new proof of a result from [20, VI.4.7], which reflects an interest (visible elsewhere in this paper, e.g. 10.1) in controlling the distribution of sets of indices. 
Definition 8.1. A first-order theory has the tree property (more precisely, the 2tree property) if there is a formula $\varphi(x ; \bar{y})$ which has the 2-tree property, where this means that in any $\aleph_{1}$-saturated model $M \models T$ there exist $\left\langle\bar{a}_{\eta}: \eta \in{ }^{\aleph_{0}>} 2\right\rangle$ such that:

(1) For $\eta \in \aleph^{\aleph_{0}>2}$ and $i, j<\omega,\left\{\varphi\left(x ; \bar{a}_{\eta-i}\right), \varphi\left(x ; \bar{a}_{\eta-j}\right)\right\}$ is inconsistent.

(2) For $\eta \in \aleph^{\aleph_{0}} 2,\left\{\varphi\left(x ; \bar{a}_{\left.\eta\right|_{i}}\right): i<\aleph_{0}\right\}$ is a consistent partial $\varphi$-type.

If $T$ has the tree property, we say it is not simple, otherwise it is simple.

We will use $\unlhd$ to indicate comparability in the tree, i.e. $\eta \unlhd \rho$ means $\eta$ is before $\rho$ in the partial tree order.

Remark 8.2. Definition 8.1 remains agnostic about whether $\left\{\varphi\left(x ; \bar{a}_{\eta}\right), \varphi\left(x ; \bar{a}_{\rho}\right)\right\}$ is consistent when $\eta, \rho$ are incomparable but not necessarily successors of the same node. Theorem $\mathrm{H}$ says, in some sense, that we may additionally assume (perhaps after changing the formula) the answer to this question is either always yes or always no.

Theorem $\mathbf{H}$ ([20, III.7.11] in our language, or [21]). If $T$ is not simple, then $T$ contains either a formula with $T P_{1}$ (equivalently $S O P_{2}$ ) or a formula with $T P_{2}$, where this means:

(1) $T$ has $T P_{1}$, or equivalently $S O P_{2}$, if there is a formula $\varphi(x ; \bar{y})$ which has $S O P_{2}$, where this means that in any $\aleph_{1}$-saturated model $M \models T$ there exist $\left\langle\bar{a}_{\eta}: \eta \in \aleph^{\aleph_{0}>} 2\right\rangle$ such that:

(a) for $\eta, \rho \in \aleph_{0}>2$ incomparable, i.e. $\neg(\eta \unlhd \rho) \wedge \neg(\rho \unlhd \eta)$, we have that $\left\{\varphi\left(x ; \bar{a}_{\eta}\right), \varphi\left(x ; \bar{a}_{\rho}\right)\right\}$ is inconsistent,

(b) for $\eta \in \aleph^{\aleph_{0}} 2,\left\{\varphi\left(x ; \bar{a}_{\left.\eta\right|_{i}}\right): i<\aleph_{0}\right\}$ is a consistent partial $\varphi$-type.

(2) $T$ has $T P_{2}$ if there is a formula $\varphi(x ; \bar{y})$ which has $T P_{2}$, where this means that in any $\aleph_{1}$-saturated model $M \models T$ there exists an array $A=\left\{\bar{a}_{j}^{i}: i<\right.$ $\omega, j<\omega\}$ of tuples, $\ell\left(\bar{a}_{j}^{i}\right)=\ell(\bar{y})$, such that: for any finite $X \subseteq \omega \times \omega$, the partial type

$$
\left\{\varphi\left(x ; \bar{a}_{j}^{i}\right):(i, j) \in X\right\}
$$

is consistent if and only if

$$
(i, j) \in X \wedge\left(i^{\prime}, j^{\prime}\right) \in X \wedge i=i^{\prime} \Longrightarrow j=j^{\prime} .
$$

Observe that the tree property is strong enough to ensure that all instances at a given level are inconsistent:

Observation 8.3. Let $T$ have the tree property and $M$ be a $\lambda^{++}$-saturated model of $T$. Then there is a formula $\varphi$ in $T$ and a sequence $\left\langle\bar{a}_{\eta}: \eta \in \lambda^{+}>\lambda\right\rangle$ of elements of $M$ such that:

(1) For $\gamma<\lambda$ and $\eta, \rho \in{ }^{\gamma} \lambda$, i.e. $\lg (\eta)=\lg (\rho),\left\{\varphi\left(x ; \bar{a}_{\eta}\right), \varphi\left(x ; \bar{a}_{\rho}\right)\right\}$ is inconsistent.

(2) For $\eta \in{ }^{\omega} 2,\left\{\varphi\left(x ; \bar{a}_{\left.\eta\right|_{i}}\right): i<\omega\right\}$ is a consistent partial $\varphi$-type.

Proof. Apply Theorem $\mathrm{H}$ and in either case, we argue by compactness that we can find such a tree for $\left\{\eta: \eta \in{ }^{n} m\right\}$ for $n, m$ finite. If some $\varphi$ has $T P_{1}$, this is immediate from the definition. Otherwise, some $\varphi$ has $T P_{2}$, so let $A$ be an array $\left\{\bar{a}_{i, j}: i<\omega, j<\omega\right\}$ as in Theorem $\underline{\mathrm{H}}(2)$. For each $k<n$, let $\left\{X_{\ell}^{k}: \ell \in{ }^{k} m\right\}$ be a partition of $\left\{\bar{a}_{k, j}: j<\omega\right\}$ into disjoint infinite sets. Then choose $\left\langle\bar{a}_{\eta}: \eta \in{ }^{n} m\right\rangle$ so that $\bar{a}_{\emptyset}=\bar{a}_{0,0}$ and for each $\eta \in{ }^{n-1} m,\left\{\bar{a}_{\eta^{-} i}: i<m\right\}$ are distinct elements of $X_{\eta}^{\lg (\eta)}$. 
Fact 8.4. Suppose that $\mathcal{D}$ is a regular ultrafilter on $\lambda, \lambda=\lambda^{<\lambda}$ or just $\left(\aleph_{1}, \aleph_{0}\right) \rightarrow$ $\left(\lambda^{+}, \lambda\right)$ (see [6]). Let $\kappa=\aleph_{0}$. Then for each $\epsilon<\lambda$ we may choose a sequence of sets $\bar{u}_{\epsilon}=\left\langle u_{\epsilon, \alpha}: \alpha<\lambda^{+}\right\rangle$such that:

(1) $u_{\epsilon, \alpha} \subseteq \alpha$.

(2) $\left|u_{\epsilon, \alpha}\right|<\lambda$.

(3) $\alpha \in u_{\epsilon, \beta} \Longrightarrow u_{\epsilon, \alpha}=u_{\epsilon, \beta} \cap \alpha$.

(4) If $u \subseteq \lambda^{+},|u|<\kappa$, then

$$
\left\{\epsilon<\lambda: \exists \alpha\left(u \subseteq u_{\epsilon, \alpha}\right)\right\} \in \mathcal{D} .
$$

Proof. By Kennedy-Shelah-Vaananen [7, p. 819] this is true when $\lambda$ satisfies the stated hypothesis and $\mathcal{D}$ is regular. Note that as briefly mentioned there, in the case of singular $\lambda$, the claim may be true; but it is also consistent that it may fail.

Claim 8.5. Suppose $\mathcal{D}$ and $\lambda$ satisfy the hypotheses of Fact 8.4. Suppose $T$ is not simple and let $M \models T$ be $\lambda^{++}$-saturated. Then $M^{\lambda} / \mathcal{D}$ is not $\lambda^{++}{ }_{\text {-saturated, }}$ and in particular is not $\lambda^{++}$-saturated for $\varphi$-types for some formula $\varphi$ with the tree property.

Proof. Let $\varphi$ and $\left\langle\bar{a}_{\eta}: \eta \in^{\lambda^{+}}>\lambda\right\rangle$ be given by Observation 8.3 , so this is a tree in the index model $M$. Let $\bar{u}$ be given by Fact 8.4 . For each $\epsilon<\lambda, \alpha<\lambda^{+}$let $\eta_{\epsilon, \alpha}$ list $u_{\epsilon, \alpha} \cup\{\alpha\}$ in increasing order. So $\eta_{\epsilon, \alpha}$ is an element of ${ }^{(\lambda>)} \lambda^{+}$, and by our choice of tree,

$$
\operatorname{otp}\left(u_{\epsilon, \alpha}\right)=\operatorname{otp}\left(u_{\epsilon, \beta}\right) \Longrightarrow\left\{\varphi\left(x, \bar{a}_{\eta_{\epsilon, \alpha}}\right), \varphi\left(x, \bar{a}_{\eta_{\epsilon, \beta}}\right)\right\} \text { is inconsistent. }
$$

From this tree in the index model, we now build an omitted type in the ultrapower. For each $\alpha<\lambda^{+}$, define a function $f_{\alpha}$ from $I$ to $\lg (y) \mathfrak{C}$ by $f_{\alpha}(\epsilon)=\bar{a}_{\eta_{\epsilon, \alpha}}$. Now, for each $\alpha_{0}<\alpha_{1}<\lambda^{+}$, the set

$$
\left\{\epsilon<\lambda:(\exists \alpha)\left(\left\{\alpha_{0}, \alpha_{1}\right\} \subseteq u_{\epsilon, \alpha}\right)\right\} \in \mathcal{D}
$$

which implies that for each such $\alpha, u_{\epsilon, \alpha} \cap \alpha_{1}=u_{\epsilon, \alpha_{1}}$. Thus, $\alpha_{0} \in u_{\epsilon, \alpha_{1}}$ on this large set. More generally, for $n<\omega$ and $\alpha_{0}<\cdots<\alpha_{n}<\lambda^{+}$, the set

$$
\left\{\epsilon<\lambda: \ell<n \Longrightarrow \alpha_{\ell} \in u_{\epsilon, \alpha_{\ell+1}}\right\} \in \mathcal{D} \text {. }
$$

Thus for any $n<\omega$ and any $\alpha_{0}<\cdots<\alpha_{n}<\lambda^{+},\left\{\epsilon<\lambda: \eta_{\epsilon, \alpha_{0}} \triangleleft \eta_{\epsilon, \alpha_{1}} \triangleleft \cdots \triangleleft \eta_{\epsilon, \alpha_{n}}\right\} \in$ $\mathcal{D}$ and therefore

$$
\left\{\epsilon<\lambda: M \models \exists x \bigwedge_{\ell} \varphi\left(x ; a_{\epsilon, \alpha_{\ell}}\right)\right\} \in \mathcal{D} .
$$

Since $n, \alpha_{0}, \ldots, \alpha_{n}$ were arbitrary, this shows

$$
p=\left\{\varphi\left(x ; f_{\alpha} / \mathcal{D}\right): \alpha<\lambda^{+}\right\}
$$

is a consistent partial type.

Assume for a contradiction that $p$ is realized, say by $f_{*} \in{ }^{\lambda} M$. For each $\alpha<\lambda^{+}$, define $J_{\alpha}=\left\{\epsilon<\lambda: M \models \varphi\left(f_{*}(\epsilon), f_{\alpha}(\epsilon)\right)\right\}$ to be the set of indices on which the supposed realization satisfies the $\alpha$ th formula of the type. We assumed $f_{*} \models p$, so $\alpha<\lambda^{+}$implies $J_{\alpha} \in \mathcal{D}$, thus $J_{\alpha} \neq \emptyset$ and we may choose some $\epsilon_{\alpha} \in J_{\alpha}$. Since $\lambda^{+}>\lambda$ is regular, there is some $\epsilon_{*}<\lambda$ such that $\left|S_{0}\right|=\lambda^{+}$, where $S_{0}=\left\{\alpha<\lambda^{+}\right.$: $\left.\epsilon_{\alpha}=\epsilon_{*}\right\} \subseteq \lambda^{+}$. As the set $\left\{\operatorname{otp}\left(u_{\epsilon, \alpha}\right): \alpha \in S_{0}\right\}$ has cardinality $\lambda$, there is $\gamma<\lambda$ such that $\left|S_{1}\right|=\lambda^{+}$, where

$$
S_{1}=\left\{\alpha \in S_{0}: \operatorname{otp}\left(u_{\epsilon, \alpha}\right)=\gamma\right\} .
$$


In particular, $\left|S_{1}\right| \geq 2$, so let $\alpha \neq \beta$ be distinct elements of $S_{1}$. Then by choice of $\epsilon_{*}, f\left(\epsilon_{*}\right)$ realizes

$$
\left\{\varphi\left(x, f_{\alpha}\left(\epsilon_{*}\right)\right), \varphi\left(x, f_{\alpha}\left(\epsilon_{*}\right)\right)\right\} \text {, i.e. }\left\{\varphi\left(x, \bar{a}_{\eta_{\epsilon_{*}, \alpha}}\right), \varphi\left(x, \bar{a}_{\eta_{\epsilon_{*}, \beta}}\right)\right\} .
$$

But (11) contradicts the consistency of (2). So $p$ is not realized, which completes the proof.

Conclusion 8.6. Let $\lambda=\lambda^{<\lambda}$ and let $\mathcal{D}$ be a regular ultrafilter on $\lambda$. If $T$ is not simple and $M \models T$, then there is $\varphi$ such that $M^{\lambda} / \mathcal{D}$ is not $\lambda^{++}$-saturated for $\varphi$-types.

Remark 8.7. Let $D_{1}, D_{2}$ be ultrafilters on $\lambda, \kappa$ respectively and suppose that $\kappa=$ $\kappa^{<\kappa}$. If $\lambda \geq \kappa^{+}$and $D_{2}$ is regular, then by Theorem E(5) and Conclusion [8.6. $D_{1} \times D_{2}$ cannot be good for equality.

\section{9. $\kappa$-COMPLETE NOT $\kappa^{+}$-COMPLETE IMPLIES NO $(\kappa, \kappa)$-CUTS}

Claim 9.1. Suppose that $\mathcal{E}$ is a $\kappa$-complete but not $\kappa^{+}$-complete ultrafilter on $I$ and $M_{1}$ is a $\kappa^{+}$-saturated model in which a linear order $L$ and tree $T$ are interpreted. Then in $M_{2}=M_{1}^{I} / \mathcal{E}$ :

(a) The linear order $L^{M_{2}}$ has no $(\kappa, \kappa)$-cut, and moreover no $(\theta, \sigma)$-cut for $\theta, \sigma<\kappa$ both regular.

(b) The tree $T^{M_{2}}$ has no branch (i.e. maximal linearly ordered set) of cofinality $\leq \kappa$.

Remark 9.2. In the statement of Claim 9.1

(1) In (a), the $\kappa$-saturation of $M_{1}$ is necessary in the following sense: if there is a sequence $\bar{\theta}=\left\langle\theta_{t}: t \in I\right\rangle$, which certainly need not be distinct, such that $M$ has a $\left(\theta_{t}, \theta_{t}\right)$-cut for each $t \in I$ and $\left(\prod_{t \in I} \theta_{t},<_{\mathcal{E}}\right)$ has cofinality $\kappa$, then the conclusion of Claim 9.1(a) is false.

(2) By this claim, we may add to the conclusion of Theorem 7.4 that $(\kappa, \kappa) \notin$ $\mathcal{C}(\mathcal{E})$ (Definition 2.13), since in that theorem the ultrafilter $E$ is a $\kappa$-complete uniform ultrafilter on $\kappa$ and thus not $\kappa^{+}$-complete.

Proof of Claim 9.1 .

(a) The "moreover" clause in (a) follows from the fact that $M_{1}$ and $M_{2}$ are $L_{\kappa, \kappa}$-equivalent, by the completeness of $\mathcal{E}$, and the hypothesis on saturation of $M_{1}$.

So we consider a potential $(\kappa, \kappa)$-cut in $M_{2}$, i.e. a $(\kappa, \kappa)$-pre-cut given by $\left\langle f_{\alpha}: \alpha<\kappa\right\rangle,\left\langle g_{\alpha}: \alpha<\kappa\right\rangle$ where if $\alpha<\beta<\kappa$, then

$$
M_{2}=\left(f_{\alpha} / \mathcal{E}\right)<_{L}\left(f_{\beta} / \mathcal{E}\right)<_{L}\left(g_{\beta} / \mathcal{E}\right)<_{L}\left(g_{\alpha} / \mathcal{E}\right) .
$$

For $0<\gamma<\kappa$ let

$$
A_{\gamma}=\left\{t: \text { if } \alpha<\beta<\gamma \text {, then } M_{1} \models f_{\alpha}(t)<_{L} f_{\beta}(t)<_{L} g_{\beta}(t)<_{L} g_{\alpha}(t)\right\} .
$$

Let $A_{0}=A_{1}=I$. Then $\bar{A}=\left\langle A_{\gamma}: \gamma<\kappa\right\rangle$ is a continuously decreasing sequence of elements of $\mathcal{E}$, i.e.:

- $\gamma_{1}<\gamma_{2} \Longrightarrow A_{\gamma_{1}} \supseteq A_{\gamma_{2}}$,

- for limit $\delta<\kappa, A_{\delta}=\bigcap\left\{A_{\gamma}: \gamma<\delta\right\}$,

- each $A_{\gamma} \in \mathcal{E}$, by choice of the functions and $\kappa$-completeness. 
As we assumed $\mathcal{E}$ is $\kappa$-complete but not $\kappa^{+}$-complete, there is a sequence $\bar{B}=$ $\left\langle B_{\gamma}: \gamma<\kappa\right\rangle$ of elements of $\mathcal{E}$ such that $\bigcap\left\{B_{\gamma}: \gamma<\kappa\right\}=\emptyset$. We may furthermore assume that $\bar{B}$ is a continuously decreasing sequence (if necessary, inductively replace $B_{\delta}$ by $\bigcap\left\{B_{\gamma}: \gamma<\delta\right\}$ using $\kappa$-completeness).

Thus given $\bar{A}, \bar{B}$, for each $t \in I$ we may define

$$
\gamma(t)=\min \left\{\alpha: t \notin A_{\alpha+1} \cap B_{\alpha+1}\right\} .
$$

By choice of $\bar{B}, t \mapsto \gamma(t)$ is a well-defined function from $I$ to $\kappa$, and $t \in A_{\gamma(t)} \cap$ $B_{\gamma(t)}$. Recall that we want to show that our given $(\kappa, \kappa)$-sequence is not a cut. Choose $f_{\kappa}, g_{\kappa} \in{ }^{I} M$ so that first, for each $t \in I, f_{\kappa}(t), g_{\kappa}(t) \in L^{M_{1}}$, and second, for each $t \in I$ and all $\alpha<\gamma(t)$,

$$
M_{1} \models f_{\alpha}(t) \leq_{L} f_{\kappa}(t)<_{L} g_{\kappa}(t) \leq_{L} g_{\alpha}(t) .
$$

This we can do by the choice of $\bar{A}$ as a continuously decreasing sequence (so the function values $f_{\alpha}, g_{\beta}$ below $\gamma(t)$ in each index model are correctly ordered) and the saturation hypothesis on $M_{1}$. Thus for each $\alpha<\kappa$, we have that

$$
\left\{t: f_{\alpha}(t) \leq_{L} f_{\kappa}(t)<_{L} g_{\kappa}(t) \leq_{L} g_{\alpha}(t)\right\} \supseteq A_{\alpha+1} \cap B_{\alpha+1} \in \mathcal{E}
$$

which completes the proof.

(b) Similar proof, but we only need to use one sequence $\left\langle f_{\alpha}: \alpha<\kappa\right\rangle$ which we choose to potentially witness that the cofinality of the branch is at most $\kappa$.

We prove a related fact for normal filters (Definition 2.6).

Claim 9.3. Assume $\mathcal{E}$ is a normal filter on $\lambda$ and $M$ is a $\lambda^{+}$-saturated dense linear order. Then $M^{I} / \mathcal{E}$ is $\lambda^{+}$-saturated.

Proof. Suppose that $\left\langle f_{\alpha} / \mathcal{E}: \alpha<\kappa_{1}\right\rangle$ is increasing in $M^{I} / \mathcal{E}$, and $\left\langle g_{\beta} / \mathcal{E}: \beta<\kappa_{2}\right\rangle$ is decreasing in $M^{I} / \mathcal{E}$, with $\kappa_{1}, \kappa_{2} \leq \lambda$ and $f_{\alpha} / \mathcal{E}<g_{\beta} / \mathcal{E}$ for $\alpha<\kappa_{1}, \beta<\kappa_{2}$. Let

$$
X_{\alpha, \beta}=\left\{t \in \lambda: f_{\alpha}(t)<g_{\beta}(t)\right\} \in \mathcal{E}
$$

for $\alpha<\beta<\lambda$. Without loss of generality, suppose $\kappa_{1} \leq \kappa_{2}$. For each $\beta<\kappa_{2}$, let

$$
Y_{\beta}=\left\{\alpha \in \lambda:(\forall j<(1+\alpha) \cap \beta)\left(j \in X_{\alpha, \beta}\right)\right\} \in \mathcal{E}
$$

by normality. For $\beta \geq \kappa_{2}$, let $Y_{\beta}=I$. Finally, define

$$
Z=\left\{\beta \in \lambda:\left(\forall k<(1+\beta) \cap \kappa_{2}\right)\left(j \in Y_{\beta}\right)\right\} \in \mathcal{E} .
$$

Now if $t \in Z$ (so $t$ plays the role of $\beta$ ) we have that

$$
p_{t}=\left\{f_{\alpha}(t)<x<g_{t}(t): \alpha<t\right\}
$$

is a consistent partial type, realized in $M$ by the saturation hypothesis. Choose $h \in{ }^{\lambda} M$ such that for each $t \in Z, h \models p_{t}$. Then $h$ realizes the type.

Note that Claim 9.3 implies by Fact $\mathbf{A . 2}$ of the Appendix that the relevant $\mathcal{E}$ is good. 


\section{0. $\mathcal{E}$ NORMAL AND $\kappa$-COMPLETE ON $\kappa$ IMPLIES A $\left(\kappa^{+}, \kappa^{+}\right)$-CUT}

The use of the additional hypothesis "normal" in this section comes in Step 3 of the proof of Claim 10.1, and consequently in later results which rely on it. Recall Definition 2.6 and Fact 2.7

Claim 10.1. Assume $\kappa$ measurable, $\mathcal{E}$ a normal $\kappa$-complete ultrafilter on $\kappa, \lambda \geq \kappa$, and $M_{1}$ a $\lambda$-saturated model with $\left(L_{M},<_{M}\right)$ a dense linear order. Let $M_{2}=M_{1}^{\kappa} / \mathcal{E}$. Then $L_{M_{2}}$ has a $\left(\kappa^{+}, \kappa^{+}\right)$-cut.

Proof. The proof has several steps.

Step 1: Fixing sequences of indices. For each $\alpha<\kappa^{+}$choose $\overline{\mathcal{U}}_{\alpha}=\left\langle u_{\alpha, \epsilon}: \epsilon<\kappa\right\rangle$ so that:

(a) This sequence is $\subseteq$-increasing and continuous, and for each $\alpha<\kappa^{+}, u_{\alpha, 0}=\emptyset$.

(b) For each $\epsilon<\kappa,\left|u_{\alpha, \epsilon}\right|<\kappa$.

(c) $\bigcup\left\{u_{\alpha, \epsilon}: \epsilon<\kappa\right\}=\alpha$.

(d) (For coherence) for $\beta<\alpha<\kappa^{+}$,

$$
\beta \in u_{\alpha, \epsilon} \Longrightarrow u_{\beta, \epsilon} \subseteq u_{\alpha, \epsilon} .
$$

Such a sequence will always exist as $|\alpha| \leq \kappa$. [Details: Clearly such a sequence exists for $\alpha \leq \kappa$ : let $u_{\kappa, \epsilon}=\epsilon \cap \alpha$, so for arbitrary $\kappa \leq \alpha<\kappa^{+}$, fixing a bijection to $\kappa$ let $\bar{V}_{\alpha}=\left\langle v_{\alpha, \epsilon}: \epsilon\langle\kappa\rangle\right.$ be the preimage of the sequence for $\kappa$. Having thus fixed, for each $\alpha<\kappa^{+}$, a sequence satisfying (a)-(c) we may then inductively pad these sequences to ensure coherence. For $\beta=0$ and each $\epsilon<\kappa$, let $u_{\beta, \epsilon}=v_{\beta, \epsilon}$. For $0<\beta<\kappa^{+}$and for each $\epsilon<\kappa$ let $u_{\beta, \epsilon}=\bigcup\left\{u_{\alpha, \epsilon}: \alpha \in v_{\beta, \epsilon}\right\}$, and note that this will preserve (b), (a), (c) and ensure (d).]

Step 2: The inductive construction of the (pre-)cut. We now construct a cut. We will first describe the construction, and then show that it is in fact a cut (i.e. we will show that we have indeed constructed a pre-cut, and that this pre-cut is not realized).

By induction on $\alpha<\kappa^{+}$we will choose $f_{\alpha}, g_{\alpha} \in{ }^{\kappa}\left(L_{M_{1}}\right)$. The intention is that each $u_{\alpha, \epsilon}$ represents a small set of prior functions which we take into account when choosing the values for $f_{\alpha}, g_{\alpha}$ at the index $\epsilon \in \kappa$.

At stage $\alpha$, for each index $\epsilon<\kappa$ define

$$
\begin{array}{cc}
w_{\alpha, \epsilon}=\left\{\beta \in u_{\alpha, \epsilon}:\right. & \left\langle f_{\gamma}(\epsilon): \gamma \in u_{\alpha, \epsilon} \cap(\beta+1)\right\rangle \text { is }<_{L\left(M_{1}\right)^{-}} \text {-increasing, } \\
& \left\langle g_{\gamma}(\epsilon): \gamma \in u_{\alpha, \epsilon} \cap(\beta+1)\right\rangle \text { is }<_{L\left(M_{1}\right)} \text {-decreasing, } \\
& \text { and } \left.f_{\beta}(\epsilon)<_{L\left(M_{1}\right)} g_{\beta}(\epsilon)\right\} .
\end{array}
$$

Our aims in defining $f_{\alpha}, g_{\alpha}$ are, on the one hand, to continue describing a precut, and on the other hand, to stay as close to the linearly ordered $w_{\alpha, \epsilon}$ as possible, as we now describe. That is, for fixed $\alpha$ for each $\epsilon$, we will choose $f_{\alpha}, g_{\alpha}$ so that:

(e) For all $\beta \in w_{\alpha, \epsilon}, M_{1} \models f_{\beta}(\epsilon)<f_{\alpha}(\epsilon)<g_{\alpha}(\epsilon)<g_{\beta}(\epsilon)$,

i.e. locally we continue the pre-cut described by $w_{\alpha, \epsilon}$.

(f) For all $\beta<\alpha$, neither $M_{1} \models f_{\beta}(\epsilon)<f_{\alpha}(\epsilon)<g_{\beta}(\epsilon)<g_{\alpha}(\epsilon)$ nor $M_{1} \models$ $f_{\alpha}(\epsilon)<f_{\beta}(\epsilon)<g_{\alpha}(\epsilon)<g_{\beta}(\epsilon)$,

i.e. the intervals are either nested or disjoint. 
(g) If $\gamma \in \alpha$ and $f_{\gamma}(\epsilon), g_{\gamma}(\epsilon)$ satisfy:

$$
\beta \in w_{\alpha, \epsilon} \Longrightarrow f_{\beta}(\epsilon)<f_{\gamma}(\epsilon)<g_{\gamma}(\epsilon)<g_{\beta}(\epsilon),
$$

then the intervals $\left[f_{\alpha}(\epsilon), g_{\alpha}(\epsilon)\right]_{L\left(M_{1}\right)},\left[f_{\beta}(\epsilon), g_{\beta}(\epsilon)\right]_{L\left(M_{1}\right)}$ are disjoint,

i.e. inside the pre-cut given by $w_{\alpha, \epsilon}$ we avoid any further refinements: we realize exactly the initial segment given by $w_{\alpha, \epsilon}$.

We will show in Step 3 that by the hypothesis on $\kappa$, (a)-(g) imply the further condition that for each fixed $\alpha<\kappa^{+}$,

(h) For all $\beta<\alpha,\left\{\epsilon: f_{\beta}(\epsilon)<f_{\alpha}(\epsilon)<g_{\alpha}(\epsilon)<g_{\beta}(\epsilon)\right\} \in \mathcal{E}$, i.e. the functions chosen will ultimately describe a pre-cut.

At each index $\epsilon<\kappa$, we may choose $f_{\alpha}(\epsilon), g_{\alpha}(\epsilon)$ to satisfy (e),(f),(g) simply because $L_{M_{1}}$ is dense and $\lambda^{+}$-saturated; the definition of $w_{\alpha, \epsilon}$ ensures (e) describes a pre-cut; and since (f) is inductively satisfied, (g) is possible.

Step 3: For $\beta<\alpha, f_{\beta}<f_{\alpha}<g_{\alpha}<g_{\beta}$. In this step we verify that for the objects constructed in the previous step, for each $\alpha<\kappa^{+}$and all $\beta<\alpha$,

$$
X_{\alpha, \beta}=\left\{\epsilon<\kappa: \beta \in u_{\alpha, \epsilon}, f_{\beta}(\epsilon)<f_{\alpha}(\epsilon)<g_{\alpha}(\epsilon)<g_{\beta}(\epsilon)\right\} \in \mathcal{E} .
$$

(By conditions (a)-(d), requiring $\beta \in u_{\alpha, \epsilon}$ does not affect membership in $\mathcal{E}$.) Suppose this is not the case, so let $\alpha<\kappa^{+}$be minimal for which there is $\beta<\alpha$ with $X_{\alpha, \beta} \notin \mathcal{E}$, and having fixed $\alpha$, let $\beta<\alpha$ be minimal such that $X_{\alpha, \beta} \notin \mathcal{E}$. For the remainder of this step we fix this choice of $\alpha, \beta$.

Since $\beta<\alpha$, by construction (that is, by (e),(f),(g) of Step 2)

$$
X_{\alpha, \beta} \subseteq\left\{\epsilon<\kappa: \beta \in u_{\alpha, \epsilon} \backslash w_{\alpha, \epsilon}\right\} .
$$

Define a function $\mathbf{x}: \kappa \rightarrow \kappa$ by

$$
\begin{aligned}
& t \mapsto \max \left\{\epsilon \leq t: \quad\left\langle f_{\gamma}(t): \gamma \in u_{\alpha, \epsilon}\right\rangle \text { is }<_{L\left(M_{1}\right)}\right. \text {-increasing, } \\
& \left\langle g_{\gamma}(t): \gamma \in u_{\alpha, \epsilon}\right\rangle \text { is }<_{L\left(M_{1}\right)} \text {-decreasing, } \\
& \text { and } \left.\gamma \in u_{\alpha, \epsilon} \Longrightarrow f_{\gamma}(t)<_{L\left(M_{1}\right)} g_{\gamma}(t)\right\} \text {. }
\end{aligned}
$$

This is well defined by Step 1, condition (a): 0 belongs to the set on the right hand side, and by continuity, there are no new conditions at limits.

For each $\epsilon<\kappa$, the set $\{t<\kappa: \mathbf{x}(t)>\epsilon\} \in \mathcal{E}$. This is because:

(1) by (c) $\left|u_{\alpha, \epsilon}\right|<\kappa$,

(2) by minimality of $\alpha$, for any $\gamma<\gamma^{\prime}<\alpha$ (e.g. any two elements of $u_{\alpha, \epsilon}$ ) we have that $X_{\gamma, \gamma^{\prime}} \in \mathcal{E}$,

(3) $\mathcal{E}$ is $\kappa$-complete,

(4) by (a) $\epsilon^{\prime}<\epsilon \Longrightarrow u_{\alpha, \epsilon^{\prime}} \subseteq u_{\alpha, \epsilon}$.

Notice that for any $t<\kappa, \mathbf{x}(t)=t$ implies $u_{\alpha, t}=w_{\alpha, t}$. So if $\mathbf{x}(t)=t$ on an $\mathcal{E}$-large set, $X_{\alpha, \beta} \in \mathcal{E}$, which would finish the proof. Suppose, then, that $Y=\{t<$ $\kappa: \mathbf{x}(t)<t\} \in \mathcal{E}$. By normality (Fact 2.7), there is $Z \subseteq Y, Z \in \mathcal{E}$ on which $\mathbf{x}(t)=\epsilon_{*}$ for some fixed $\epsilon_{*}<\kappa$. But this contradicts the first sentence of the previous paragraph.

These contradictions prove that for no $\alpha, \beta$ can it happen that $X_{\alpha, \beta} \notin \mathcal{E}$, which finishes the proof of Step 3. 
Step 4: The pre-cut is not realized, i.e. it is indeed a cut. In this step we assume, for a contradiction, that there is $h \in{ }^{\kappa} M_{1}$ such that for each $\alpha<\kappa^{+}$

$$
f_{\alpha} / \mathcal{E}<_{L} h / \mathcal{E}<_{L} g_{\alpha} / \mathcal{E},
$$

i.e. $h$ realizes the type. Fixing such an $h$, let

$$
A_{\alpha}=\left\{\epsilon<\kappa: f_{\alpha}(\epsilon)<_{L} h(\epsilon)<_{L} g_{\alpha}(\epsilon)\right\} \in \mathcal{E} .
$$

Since to each $\alpha$ we may associate a choice of index in $A_{\alpha}$, by Fodor's lemma for some $\epsilon_{*}<\kappa$,

$$
S_{1}=\left\{\delta: \delta<\kappa^{+}, \operatorname{cf}(\delta)=\kappa, \epsilon_{*} \in A_{\delta}\right\}
$$

is stationary. Furthermore, since $\left|u_{\epsilon_{*}, \alpha}\right|<\kappa$, there is some $w_{*} \subseteq u_{\epsilon, \alpha}$ for which

$$
S_{2}=\left\{\delta \in S_{1}: w_{\epsilon_{*}, \delta}=w_{*}\right\} \subseteq \kappa^{+}
$$

is stationary. Let $\delta_{*} \in S_{2}$ be such that $\left|\delta_{*} \cap S_{2}\right|=\kappa$. As $\left|w_{\epsilon_{*}, \delta_{*}}\right| \leq\left|u_{\epsilon_{*}, \delta_{*}}\right|<\kappa$, we may choose $\gamma_{*} \in S_{2} \cap\left\{\delta_{*} \backslash w_{\epsilon_{*}, \delta_{*}}\right\}$.

Now $w_{\epsilon_{*}, \delta_{*}}=w_{\epsilon_{*}, \gamma_{*}}=w_{*}$ since $\delta_{*}, \gamma_{*} \in S_{2}$, and note $\gamma_{*}<\delta_{*}$. The definition of the sets $w$ (here, $w_{*}$ ) and Step 3, condition (e), means that when choosing $f_{\delta_{*}}\left(\epsilon_{*}\right), g_{\delta_{*}}\left(\epsilon_{*}\right)$ we would have ensured that

$$
\beta \in w_{*} \Longrightarrow f_{\beta}\left(\epsilon_{*}\right)<f_{\delta_{*}}\left(\epsilon_{*}\right)<g_{\delta_{*}}\left(\epsilon_{*}\right)<g_{\beta}\left(\epsilon_{*}\right)
$$

and likewise that

$$
\beta \in w_{*} \Longrightarrow f_{\beta}\left(\epsilon_{*}\right)<f_{\gamma_{*}}\left(\epsilon_{*}\right)<g_{\gamma_{*}}\left(\epsilon_{*}\right)<g_{\beta}\left(\epsilon_{*}\right) .
$$

On the other hand, $\gamma_{*}<\delta_{*}$ and $\gamma_{*} \notin w_{*}$. So when choosing $f_{\gamma_{*}}(\epsilon), g_{\gamma_{*}}(\epsilon)$, Step 3 , condition $(\mathrm{g})$, would have meant we chose the intervals $\left[f_{\delta_{*}}\left(\epsilon_{*}\right), g_{\delta_{*}}\left(\epsilon_{*}\right)\right]_{L\left(M_{1}\right)}$, $\left[f_{\gamma_{*}}\left(\epsilon_{*}\right), g_{\gamma_{*}}\left(\epsilon_{*}\right)\right]_{L\left(M_{1}\right)}$ to be disjoint.

But we also know that $\gamma_{*}, \delta_{*} \in S_{1}$, so $h\left(\epsilon_{*}\right)$ must belong to both intervals. This contradiction completes Step 4 and the proof.

Remark 10.2. We know that if $D$ is any ultrafilter on $\kappa$ and $M$ is a model whose theory is not simple, then $M^{\kappa} / \mathcal{D}$ is not $\kappa^{++}$-saturated. Still, Claim 10.1 gives more precise information about the size of the cut: we are guaranteed a cut of type $\left(\kappa^{+}, \kappa^{+}\right)$as opposed to e.g. $\left(\kappa^{+}, \kappa\right)$. On the importance of symmetric cuts, see [17.

Claim 10.3. Assume $\kappa$ measurable, $\mathcal{E}$ a $\kappa$-complete filter on $\kappa, \lambda \geq \kappa$, and $M_{1} a$ $\lambda$-saturated model with $\left(L_{M},<_{M}\right)$ a dense linear order. Let $M_{2}=M_{1}^{\kappa} / \mathcal{E}$. Then $L_{M_{2}}$ has no $(\theta, \sigma)$-cut with $\theta<\kappa$ and $\sigma<\lambda$.

Proof. Suppose for a contradiction that there were such a cut given by $\left\langle f_{\alpha}: \alpha<\theta\right\rangle$, $\left\langle g_{\beta}: \beta<\sigma\right\rangle$ with $\alpha_{1}, \alpha_{2}<\theta, \beta_{1}, \beta_{2}<\sigma \Longrightarrow\left\{t: M_{1} \models f_{\alpha_{1}}(t)<_{L} f_{\alpha_{2}}(t)<_{L}\right.$ $\left.g_{\alpha_{2}}(t)<_{L} g_{\alpha_{1}}(t)\right\} \in \mathcal{E}$. Expand the language to add constants $\left\{c_{\alpha}: \alpha<\theta\right\}$ where in the $t$ th copy of the index model $M_{1}$, denoted $M_{1}[t], c_{\alpha}$ is interpreted as $f_{\alpha}(t)$. Then in the ultrapower (which in the expanded language is an ultraproduct), $\left\langle c_{\alpha}: \alpha<\theta\right\rangle$ forms the lower half of the supposed cut. For each $\beta<\sigma$, the set

$$
\left.A_{\beta}:=\bigcap\left\{t: M_{1}[t] \models c_{\alpha}<_{L} g_{\beta}[t]\right\}: \alpha<\theta\right\} \in \mathcal{E}
$$

by $\kappa$-completeness.

But recall that $M_{1}$ is a $\lambda$-saturated model, and $\sigma<\lambda$. Since for each $t$, we have

$$
\left|\left\{\beta<\sigma: t \in A_{\beta}\right\}\right| \leq \sigma<\lambda
$$


we may choose a new element $h \in{ }^{I} M_{1}$ so that for each $t, h(t)$ satisfies $\alpha<\theta \Longrightarrow$ $M_{1}[t] \models c_{\alpha}<_{L} h(t)$ and $t \in A_{\beta} \Longrightarrow M_{1}[t] \models h(t)<_{L} g_{\beta}(t)$. By Eos' theorem $h$ realizes our cut, which is the desired contradiction.

In a forthcoming paper the authors have shown that:

Theorem I (Malliaris and Shelah [17). If $\mathcal{D}$ is a regular ultrafilter on $\lambda$ which saturates some theory with $\mathrm{SOP}_{2}$, and $M$ is a model of linear order, then among other things:

(1) for all $\mu \leq \lambda, M^{\lambda} / \mathcal{D}$ has no $(\mu, \mu)$-cut,

(2) for all $\mu \leq \lambda$, there is at most one $\rho \leq \lambda$ such that $M^{\lambda} / \mathcal{D}$ has a $(\mu, \rho)$-cut.

Conclusion 10.4. Let $\kappa<\lambda$ and suppose $\kappa$ is measurable. Then there exists a regular ultrafilter $\mathcal{D}$ on $I,|I|=\lambda$ which is flexible but not good, specifically not good for any theory with $S O P_{2}$.

Proof. Let $D$ be a $\lambda^{+}$-good, $\lambda$-regular ultrafilter on $\lambda$. Let $\mathcal{E}$ be a normal $\kappa$ complete, not $\kappa^{+}$-complete ultrafilter on $\kappa$. Let $\mathcal{D}=D \times \mathcal{E}$ be the product ultrafilter. Then $\mathcal{D}$ is flexible by Corollary 7.3 . On the other hand, by Claim 10.1, any $\mathcal{D}$-ultrapower of linear order will omit a $\left(\kappa^{+}, \kappa^{+}\right)$-cut. By Theorem 【. $\mathcal{D}$ cannot saturate any theory with $\mathrm{SOP}_{2}$.

Remark 10.5. On one hand, the advantage of Conclusion 10.4 over Theorem 7.4 is in the greater range of cardinals: we ask only that $\kappa<\lambda$, not $2^{\kappa} \leq \lambda$. On the other hand, Theorem 7.4 gives an a priori stronger failure of goodness, since the random graph is minimum among unstable theories in Keisler's order.

\section{Finite Alternations of Symmetric Cuts}

In this section we iterate the results of $\S 99$, 10 to produce regular ultrafilters $\mathcal{D}$ whose library of cuts, $\mathcal{C}(\mathcal{D})$, contains any fixed finite number of alternations (or gaps). The following definition is stated for regular ultrafilters only so that the choice of index model will not matter.

Definition 11.1. Let $\kappa$ be a cardinal. Say that the regular ultrafilter $\mathcal{D}$ on $\lambda \geq \aleph_{0}$ has $\kappa$ alternations of cuts if there exist cardinals $\left\langle\mu_{\ell}: \ell<\kappa\right\rangle,\left\langle\rho_{\ell}: \ell<\kappa\right\rangle$ such that:

- $\ell_{1}<\ell_{2}<\kappa \Longrightarrow \aleph_{0}<\rho_{\ell_{1}}<\mu_{\ell_{1}}<\rho_{\ell_{2}}<\mu_{\ell_{2}}<\lambda$,

- for each $0 \leq \ell<\kappa,\left(\rho_{\ell}, \rho_{\ell}\right) \in \mathcal{C}(\mathcal{D})$, i.e. $(\mathbb{N},<)^{\lambda} / \mathcal{D}$ has some $\left(\rho_{\ell}, \rho_{\ell}\right)$-cut,

- for each $0 \leq \ell<\kappa,\left(\mu_{\ell}, \mu_{\ell}\right) \notin \mathcal{C}(\mathcal{D})$, i.e. $(\mathbb{N},<)^{\lambda} / \mathcal{D}$ has no $\left(\mu_{\ell}, \mu_{\ell}\right)$-cut.

We will start by proving a theorem for products of complete ultrafilters (Theorem 11.3), and then extend it to regular ones in Theorem 11.4 by adding one more iteration of the ultrapower.

First we observe that taking ultrapowers will not fill symmetric cuts whose cofinality is larger than the size of the index set.

Observation 11.2. Suppose $M$ is a $\lambda$-saturated model of linear order, $\kappa<\lambda, D$ an ultrafilter on $\kappa$. If $M$ contains $a\left(\kappa_{*}, \kappa_{*}\right)$-cut, where $\kappa_{*}=\operatorname{cf}\left(\kappa_{*}\right)>\kappa$, then $M^{\kappa} / D$ will also contain a $\left(\kappa_{*}, \kappa_{*}\right)$-cut. More precisely, the image of the cut from $M$ under the diagonal embedding will remain unrealized in $M^{\kappa} / D$. 
Proof. Let the cut in $M$ be given by $\left(\left\langle f_{\alpha}: \alpha<\kappa_{*}\right\rangle,\left\langle g_{\beta}: \beta<\kappa_{*}\right\rangle\right)$, and we consider the pre-cut given by $\left(\left\langle f_{\alpha} / D: \alpha<\kappa_{*}\right\rangle,\left\langle g_{\beta} / D: \beta<\kappa_{*}\right\rangle\right)$ in the ultrapower $M^{\kappa} / D$. Suppose for a contradiction that there were a realization $h \in{ }^{\kappa} M$. Let $\mathbf{x}: \kappa_{*} \rightarrow \kappa$ be a function which to each $\alpha<\kappa_{*}$ associates some index $\epsilon<\kappa$ for which $M \models f_{\alpha}(\epsilon)<h(\epsilon)<g_{\alpha}(\epsilon)$. By Fodor's lemma, there is a stationary subset $X \subseteq \kappa_{*}$ on which $\mathbf{x}$ is constant and equal to, say, $\epsilon_{*}$. Then in $M,\left(\left\langle f_{\alpha}\left(\epsilon_{*}\right): \alpha \in\right.\right.$ $\left.X\rangle,\left\langle g_{\beta}\left(\epsilon_{*}\right): \beta \in X\right\rangle\right)$ will be cofinal in the original cut, but by choice of $X$ it will be realized by $h\left(\epsilon_{*}\right)$, a contradiction.

Since the proof of Theorem 11.3 involves an inductive construction, it will be convenient to index the cardinals $\kappa_{\ell}$ in reverse order of size.

Theorem 11.3. Suppose that we are given:

(a) $n<\omega$ and $\kappa_{n}<\cdots<\kappa_{0}<\kappa_{-1}=\lambda$.

(b) $\mathcal{E}_{\ell}$ a normal $\kappa_{\ell}$-complete ultrafilter on $\kappa_{\ell}$, for $\ell \leq n$.

(c) $M_{0} a \lambda$-saturated model which is, or contains, a dense linear order $<$.

(d) $M_{\ell+1}=\left(M_{\ell}\right)^{\kappa \ell} / \mathcal{E}_{\ell}$ for $\ell \leq n$.

Then:

( $\alpha)$ For $\ell \leq n, M_{\ell+1}$ is $\kappa_{\ell}$-saturated.

( $\beta)$ If $\ell<k \leq n+1$, then $M_{k}$ has a $\left(\kappa_{\ell}{ }^{+}, \kappa_{\ell}{ }^{+}\right)$-cut.

$(\gamma)$ For $i, \ell \leq n+1, M_{\ell}$ has no $\left(\kappa_{i}, \kappa_{i}\right)$-cut.

$(\delta)$ For $\ell \leq n+1, M_{\ell}$ has no $(\theta, \theta)$-cut for $\theta<\lambda$ weakly compact.

Thus, for each $\ell \leq n,\left(\kappa_{\ell}{ }^{+}, \kappa_{\ell}{ }^{+}\right) \in \mathcal{C}\left(M_{n+1}\right)$, and for any weakly compact $\theta<\lambda$, in particular $\theta=\kappa_{\ell},(\theta, \theta) \notin \mathcal{C}\left(M_{n+1}\right)$.

Proof. The "thus" clause summarizes $(\alpha)-(\delta)$. Recall that for transparency all languages are countable. Note that condition (b) implies the cardinals $\kappa_{\ell}$ are measurable cardinals, thus limit cardinals, so condition $(\gamma)$ can never contradict condition $(\beta)$.

$(\alpha)$ By induction on $-1 \leq \ell \leq n$ we verify that $M_{\ell+1}$ is $\kappa_{\ell}$-saturated. For $\ell=-1$, $M_{0}$ is $\lambda$-saturated and $\lambda>\kappa_{0}$. For $\ell>-1$, use the fact that $M_{\ell+1}=\left(M_{\ell}\right)^{\kappa_{\ell}} / \mathcal{E}_{\ell}$ thus $M_{\ell+1} \equiv_{L_{\infty, \kappa_{\ell}}} M_{\ell}$ by Los' theorem for $L_{\kappa_{i}, \kappa_{i}}$.

$(\beta)$ By Claim 10.1 and Observation 11.2

$(\gamma)$ Follows from $(\delta)$ as measurable implies weakly compact.

$(\delta)$ We prove this by induction on $\ell \leq n$. For $\ell=-1, M_{0}$ is $\lambda$-saturated. For $\ell>-1$, let $\theta<\lambda$ be given and suppose we have a pre-cut in $M_{\ell+1}$ given by $\left(\left\langle f_{\alpha}: \alpha<\theta\right\rangle,\left\langle g_{\beta}: \beta<\theta\right\rangle\right)$. There are three cases. If $\theta<\kappa_{\ell}$, then use $(\alpha)$. If $\theta=\kappa_{\ell}$, use Claim 9.1. So we may assume $\kappa_{\ell}<\theta$. Since $\theta$ is weakly compact, therefore inaccessible, $2^{\kappa_{\ell}}<\theta$. For $\alpha<\beta<\theta$ let

$$
A_{\alpha, \beta}=\left\{\epsilon<\kappa_{\ell}: f_{\alpha}(\epsilon)<f_{\beta}(\epsilon)<g_{\beta}(\epsilon)<g_{\alpha}(\epsilon)\right\} \in \mathcal{E}_{\ell} .
$$

As $\theta$ is weakly compact, by Fact 2.5 the function $\mathbf{x}: \theta \times \theta \rightarrow 2^{\kappa_{\ell}}<\theta$ is constant on some $\mathcal{U} \in[\theta]^{\theta}$. Call this constant value $A_{*}$. Now for $\epsilon \in A_{*}$, the sequence $\left(\left\langle f_{\alpha}(\epsilon): \alpha \in \mathcal{U}\right\rangle,\left\langle g_{\beta}(\epsilon): \beta \in \mathcal{U}\right\rangle\right)$ is a pre-cut in $M_{\ell}$, meaning that $\alpha<\beta \in \mathcal{U} \Longrightarrow$ $f_{\alpha}(\epsilon)<f_{\beta}(\epsilon)<g_{\beta}(\epsilon)<g_{\alpha}(\epsilon)$.

Let $B_{*}=\left\{\epsilon \in A_{*}\right.$ : in $M_{\ell}$ there is $c$ such that $\left.\alpha \in \mathcal{U} \Longrightarrow f_{\alpha}(\epsilon)<_{M_{\ell}} c<_{M_{\ell}} g_{\alpha}(\epsilon)\right\}$. Now if $A_{*} \backslash B_{*} \neq \emptyset$, for any $\epsilon \in A_{*} \backslash B_{*}$ we have that $\left(\left\langle f_{\alpha}(\epsilon): \alpha \in \mathcal{U}\right\rangle,\left\langle g_{\beta}(\epsilon): \beta \in \mathcal{U}\right\rangle\right)$ is not just a pre-cut but also a cut in $M_{\ell}$, contradicting the inductive hypothesis. Thus for every $\epsilon \in A_{*}$ we may choose a realization $c(\epsilon)$ of the relevant pre-cut. 
For $\epsilon \in \kappa \backslash A_{*}$, let $c(\epsilon)$ be arbitrary. Then $\left\langle c(\epsilon): \epsilon<\kappa_{\ell}\right\rangle / \mathcal{E}_{\ell} \in M_{\ell+1}$ realizes $\left(\left\langle f_{\alpha}: \alpha<\theta\right\rangle,\left\langle g_{\beta}: \beta<\theta\right\rangle\right)$, as desired.

By appending the construction of Theorem 11.3 to a suitable regular ultrafilter, we may produce regular ultrapowers with $n$ alternations of cuts for any finite $n$.

Theorem 11.4. Let $\lambda$ be an infinite cardinal, $n<\omega$, and suppose that there exist measurable cardinals $\kappa_{n}<\cdots<\kappa_{0}<\lambda$. Then there is a regular ultrafilter $\mathcal{D}$ on $I$, $|I|=\lambda$ such that:

(1) $\left(\kappa_{\ell}{ }^{+}, \kappa_{\ell}{ }^{+}\right) \in \mathcal{C}(\mathcal{D})$ for $\ell \leq n$.

(2) $(\theta, \theta) \notin \mathcal{C}(\mathcal{D})$ for $\theta<\lambda$ weakly compact, in particular $\ell \leq n, \theta=\kappa_{\ell}$.

(3) $\mathcal{D}$ is $\kappa_{n}{ }^{+}$-good.

(4) $\mathcal{D}$ is $\lambda$-flexible.

(5) $\mathcal{D}$ is $\lambda^{+}$-good for countable stable theories.

(6) $\mathcal{D}$ is not $\left(2^{\kappa_{n}}\right)^{+}$-good for unstable theories.

Proof. Let $\mathcal{D}_{1}$ be a $\lambda$-regular, $\lambda^{+}$-good ultrafilter on $\lambda$. Let $\mathcal{E}$ be the ultrafilter on $\kappa_{n}$ given by $\mathcal{E}_{0} \times \cdots \times \mathcal{E}_{n}$, where the $\mathcal{E}_{\ell}$ are as in the statement of Theorem 11.3 . Let $\mathcal{D}=\mathcal{D}_{1} \times \mathcal{E}$. We will show that $\mathcal{D}$ has the desired properties.

(1)-(2) This follows from having chosen $M_{0}$ in Theorem 11.3 to be a $\mathcal{D}_{1}$-ultrapower, thus $\lambda^{+}$-saturated by the $\lambda^{+}$-goodness (and regularity) of $\mathcal{D}_{1}$.

(3) By Observation A.1.

(4) By induction on $\ell \leq n$, using Corollary [7.3(1) and the completeness of $\mathcal{E}_{\ell}$, $\ell \leq n$.

(5) By induction on $\ell \leq n$, using Corollary [7.3)(2). Since $\mathcal{D}_{1}$ is regular and $\lambda^{+}$-good, $\operatorname{lcf}\left(\aleph_{0}, \mathcal{D}\right) \geq \lambda^{+}$.

(6) By Claim 5.2

Question 11.5. Can Theorem 11.4 be generalized to any number of alternations, not necessarily finite?

\section{APPENDIX}

In this appendix, we collect several known facts, easy proofs or extensions of proofs relevant to the material in the paper.

Observation A.1. (1) Any regular ultrafilter is uniform.

(2) In the definition of good ultrafilter (Definition 1.4), we may assume the functions are monotonic.

(3) If $\mathcal{E}$ is a $\kappa$-complete ultrafilter on $\kappa$, then $\mathcal{E}$ is $\kappa^{+}$-good.

Proof. (1) Keisler [5, Theorem 1.1].

(2) By induction on $|u|$, replace a given function $f$, not necessarily monotonic, by its monotonic refinement $f^{\prime}$ given by $f^{\prime}(u)=f(u) \cap \bigcap_{v \subsetneq u} f^{\prime}(v)$.

(3) Adapt the proof of [20, Claim 3.1, p. 334] that any ultrafilter is $\aleph_{1}$-good.

Note, however, that while a good regular ultrafilter produces saturated ultrapowers, this need not be the case when the ultrafilter is complete, unless the index models are also saturated. That is, the goodness of the ultrafilter $D$ on $I$ is equivalent to saturation of the ultrapower $M^{I} / D$ when (a) we have regularity of the ultrafilter, or (b) we assume the model $M$ is saturated. 
Fact A.2. Let $D$ be an ultrafilter on $I$ and $\lambda$ a cardinal. Then the following are equivalent:

(1) $D$ is $\lambda$-good.

(2) For any model $M$ in a countable signature which is $\lambda$-saturated, $M^{I} / D$ is $\lambda$-saturated.

If $D$ is regular, (1) is equivalent to:

(3) For any model $M$ in a countable signature, $M^{I} / D$ is $\lambda$-saturated.

Proof. See [20, VI.2], in particular Theorems 2.2-2.3, Claim 2.4, and Lemma 2.11.

By representing the proof of [20, Theorem VI.4.8, p. 379] to emphasize the role of incompleteness, we obtain a more general result.

Theorem A.3. Let $M$ be a $\lambda^{+}$-saturated model of an unstable theory $T, \varphi$ an unstable formula, and $E$ a $\kappa$-complete, $\kappa^{+}$-incomplete ultrafilter on $\lambda$. Let $\delta=$ $\operatorname{lcf}(\kappa, \mathcal{E})$. Then $M^{I} / \mathcal{E}$ is not $(\kappa+\operatorname{lcf}(\kappa, \mathcal{E}))^{+}$-saturated for $\varphi$-types.

Proof. First consider the countably incomplete case. We build a correspondence between a $\varphi$-type and a <-type in an expanded language.

Let $\varphi=\varphi(x ; y)$, where without loss of generality $\ell(x)=1$ but $\ell(y)$ need not be 1 . Choose, for each $m<n<\omega$, sequences $\bar{a}_{m}^{n}$ where $\ell\left(\bar{a}_{m}^{n}\right)=\ell(y)$, so that $\varphi$ has the order property over each $\left\langle\bar{a}_{m}^{n}: m<n\right\rangle$ : i.e. $k<n<\omega$ implies $\left\{\varphi\left(x ; \bar{a}_{m}^{n}\right)^{i f(m>k)}\right.$ : $m<n\}$ is consistent.

Let $\left\langle b_{n}: n<\omega\right\rangle$ be a sequence of distinct elements. Let $P$ be a new unary relation symbol, $<$ a new binary relation symbol, and for each $\ell<\ell(y)$ let $F_{\ell}$ be a new binary function symbol. Let $M_{1}$ denote the expansion of $M$ by these new symbols, as follows. $P^{M_{1}}=\left\{b_{n}: n<\omega\right\}$ and $<^{M_{1}}=\left\{\left\langle b_{k}, b_{n}\right\rangle: k<n<\omega\right\}$. Finally, interpret the functions $F_{\ell}$ so that for each $k<n<\omega, \bar{a}_{k}^{n}=\left\langle F_{0}\left(b_{k}, b_{n}\right), \ldots, F_{\ell(y)-1}\left(b_{k}, b_{n}\right)\right\rangle$.

Now we take the ultrapower $N=M^{\lambda} / E$ and let $N_{1}$ denote the corresponding ultrapower in the expanded language. In $N_{1}, P$ is a linear order and so by the hypothesis on $E$ we have a $\delta$-cut over the diagonal embedding of the sequence $\left\langle b_{n}: n<\omega\right\rangle$. Choose a sequence $\left\langle c_{i}: i<\delta\right\rangle$ witnessing this, so (1) each $c_{i} \in P^{N_{1}}$, (2) $n<\omega$ and $i<j<\delta$ implies $N_{1} \models b_{n}<c_{i}<c_{j}$, (3) for no $c \in P^{N_{1}}$ is it the case that for each $n<\omega, i<\delta, N_{1} \mid=b_{n}<c<c_{i}$.

We now translate back to a $\varphi$-type. Consider:

$$
\begin{aligned}
q(x)= & \left\{\neg \varphi\left(x, F_{0}\left(b_{n}, c_{0}\right), \ldots, F_{\ell(y)-1}\left(b_{n}, c_{0}\right)\right): n<\omega\right\} \\
& \cup\left\{\varphi\left(x, F_{0}\left(c_{i}, c_{0}\right), \ldots, F_{\ell(y)-1}\left(c_{i}, c_{0}\right)\right): 0<i<\delta\right\} .
\end{aligned}
$$

This is a consistent partial $\varphi$-type by Łos' theorem, since for $n<\omega, i<\delta$ we have that $b_{n}<c_{i} \bmod E$. We will show that $q$ is omitted. Suppose it were realized, say, by $a$. Let $\left\langle X_{n}: n<\omega\right\rangle$ be a sequence of elements of $E$ witnessing that $E$ is $\aleph_{1}$-incomplete. Let $\left\langle Y_{n}: n<\omega\right\rangle$ be a sequence of elements of $E$ given by

$$
Y_{n}=\left\{t \in \lambda: M \models \bigwedge\left\{\neg \varphi\left(a[t], F_{0}\left(b_{k}, c_{0}[t]\right), \ldots, F_{\ell(y)-1}\left(b_{k}, c_{0}[t]\right)\right): k \leq n\right\}\right\}
$$

which exists by Los' theorem. (We write $b_{k}$ rather than $b_{k}[t]$ since these are essentially constant elements. Note that in each index model, $c_{0}[t]$ is simply one of the $b_{n}$ 's.)

For each $t \in I$ define $\rho(t)=\min \left\{n: t \notin X_{n+1} \cap Y_{n+1}\right\}$. Then $\rho$ is well defined by $\aleph_{1}$-incompleteness. Define $b \in{ }^{\lambda} M$ by: $b[t]=b_{\rho(t)}$. By Łos' theorem $P^{N_{1}}(b)$. 
Where does it fit under $<^{N_{1}}$ ? First, for each $n<\omega$, we have $b>b_{n} \bmod E$, as $E$ is $\omega$-complete (i.e. a filter) thus:

$$
\left\{t: c[t]>b_{n}[t]\right\} \supseteq \bigcap_{j \leq n}\left(X_{j+1} \cap Y_{j+1}\right) \in \mathcal{D} .
$$

On the other hand, suppose that for some $i<\delta$ we had $c_{i} \leq b \bmod E$. Then by Łos' theorem and the definition of $\rho$, it would have to be the case that

$$
N_{1} \models \neg \varphi\left(a[t], F_{0}\left(c_{i}[t], c_{0}[t]\right), \ldots, F_{\ell(y)-1}\left(c_{i}[t], c_{0}[t]\right)\right)
$$

contradicting the definition of $q$. So for each $i<\delta$, we have that $b<c_{i} \bmod E$.

Thus from a realization $a \models q$ we could construct a realization $b$ of the $\left(\aleph_{0}, \delta\right)$-cut in $P^{N}$. Since the latter is omitted, $q$ must be as well, which completes the proof for countably incomplete filters.

Now for the general case: If $\lambda=\kappa$, modify the argument of Theorem A.3 by replacing $\omega$ with $\kappa$ everywhere in the proof just given, and Łos' theorem by Łos' theorem for $L_{\kappa, \kappa}$. If $\lambda>\kappa$, begin by choosing a surjective map $\mathbf{h}: \lambda \rightarrow \kappa$ so that $\mathcal{E}=h(E)$ is a non-principal ultrafilter on $\kappa$, thus $\kappa$-complete not $\kappa^{+}$-complete.

\section{ACKNOWLEDGEMENTS}

The authors thank Simon Thomas for very helpful organizational remarks. The authors also thank the anonymous referee for many helpful comments on the paper.

\section{REFERENCES}

[1] Joni Baker and Kenneth Kunen, Limits in the uniform ultrafilters, Trans. Amer. Math. Soc. 353 (2001), no. 10, 4083-4093, DOI 10.1090/S0002-9947-01-02843-4. MR.1837221 (2002j:54025)

[2] C. C. Chang and H. J. Keisler, Model Theory, North-Holland, 1973.

[3] Alan Dow, Good and OK ultrafilters, Trans. Amer. Math. Soc. 290 (1985), no. 1, 145-160, DOI 10.2307/1999788. MR787959 (86f:54044)

[4] H. Jerome Keisler, Good ideals in fields of sets, Ann. of Math. (2) 79 (1964), 338-359. MR.0166105 (29 \#3383)

[5] H. Jerome Keisler, Ultraproducts which are not saturated, J. Symbolic Logic 32 (1967), 23-46. MR0218224 (36 \#1312)

[6] Juliette Kennedy and Saharon Shelah, On regular reduced products, J. Symbolic Logic 67 (2002), no. 3, 1169-1177, DOI 10.2178/jsl/1190150156. MR1926605(2003k:03047)

[7] Juliette Kennedy, Saharon Shelah, and Jouko Väänänen, Regular ultrafilters and finite square principles, J. Symbolic Logic 73 (2008), no. 3, 817-823, DOI 10.2178/jsl/1230396748. MR2444269(2009h:03040)

[8] Kenneth Kunen, Ultrafilters and independent sets, Trans. Amer. Math. Soc. 172 (1972), 299-306. MR0314619 (47 \#3170)

[9] Kenneth Kunen, Weak P-points in $\mathbf{N}^{*}$, Topology, Vol. II (Proc. Fourth Colloq., Budapest, 1978), Colloq. Math. Soc. János Bolyai, vol. 23, North-Holland, Amsterdam, 1980, pp. 741749. MR:588822(82a:54046)

[10] Jerzy Łoś, Quelques remarques, théorèmes et problèmes sur les classes définissables d'algèbres (French), Mathematical interpretation of formal systems, North-Holland Publishing Co., Amsterdam, 1955, pp. 98-113. MR0075156(17,700d)

[11] M. Malliaris, Realization of $\phi$-types and Keisler's order, Ann. Pure Appl. Logic 157 (2009), no. 2-3, 220-224, DOI 10.1016/j.apal.2008.09.008. MR2499710(2010b:03044)

[12] M. Malliaris, Ph. D. thesis, U.C. Berkeley (2009).

[13] M. Malliaris, Hypergraph sequences as a tool for saturation of ultrapowers, J. Symbolic Logic 77 (2012), no. 1, 195-223, DOI 10.2178/jsl/1327068699. MR2951637

[14] M. Malliaris, Independence, order, and the interaction of ultrafilters and theories, Ann. Pure Appl. Logic 163 (2012), no. 11, 1580-1595, DOI 10.1016/j.apal.2011.12.010. MR2959661 
[15] M. Malliaris and S. Shelah, Model-theoretic properties of ultrafilters built by independent families of functions, math.LO/1208.2579.

[16] M. Malliaris and S. Shelah, A dividing line within simple unstable theories, Adv. Math. 249 (2013), 250-288, DOI 10.1016/j.aim.2013.08.027. MR3116572

[17] M. Malliaris and S. Shelah, Cofinality spectrum problems in model theory, set theory and general topology, math.LO/1208.5424.

[18] M. Malliaris and S. Shelah, General topology meets model theory, on $\mathfrak{p}$ and $\mathfrak{t}$, Proc. Natl. Acad. Sci. USA 110 (2013), no. 33, 13300-13305, DOI 10.1073/pnas.1306114110. MR3105597

[19] S. Shelah, Classification Theory, North-Holland, 1978.

[20] S. Shelah, Classification theory and the number of nonisomorphic models, 2nd ed., Studies in Logic and the Foundations of Mathematics, vol. 92, North-Holland Publishing Co., Amsterdam, 1990. MR1083551 (91k:03085)

[21] S. Shelah, Simple unstable theories, Ann. Math. Logic 19 (1980), no. 3, 177-203, DOI 10.1016/0003-4843(80)90009-1. MR595012 (82g:03055)

[22] Saharon Shelah, Toward classifying unstable theories, Ann. Pure Appl. Logic 80 (1996), no. 3, 229-255, DOI 10.1016/0168-0072(95)00066-6. MR.1402297(97e:03052)

[23] S. Shelah and A. Usvyatsov, More on $\mathrm{SOP}_{1}$ and $\mathrm{SOP}_{2}$, Ann. Pure Appl. Logic 155 (2008), no. 1, 16-31, DOI 10.1016/j.apal.2008.02.003. MR2454629(2009k:03045)

Department of Mathematics, University of Chicago, 5734 S. University Avenue, Chichag, Illinois 60637

E-mail address: mem@math.uchicago.edu

Einstein Institute of Mathematics, Edmond J. Safra Campus, Givat Ram, The Hebrew University of Jerusalem, Jerusalem, 91904, Israel - And - Department of Mathematics, Hill Center - Busch Campus, Rutgers, The State University of New Jersey, 110 Frelinghuysen Road, Piscataway, New Jersey 08854-8019

E-mail address: shelah@math.huji.ac.il

URL: http://shelah.logic.at 